\title{
No. X.-LES ESPĖCES D'ALPHEIDE RAPPORTÉES DE L'OCÉAN INDIEN PAR M. J. STANLEY GARDINER.
}

\author{
Par M. le Professeur H. Coutière.
}

(Communiqué par M. le Professeur J. Stanley Gardiner, M.A., F.R.S., F.L.S.)

(Planches 60-64.)

Lu le 20 Juin, 1918.

Les espèces nouvelles d'Alpheidæ rapportées par le Percy Sladen Trust Expédition ont été décrites en 1908, dans le B. de la Soc. Philomathique. Depuis, le très important mémoire de de Man, consacré aux récoltes du Siboga, est venu montrer, surabondamment, quel fonds on peut faire sur les Alpheidæ pour caractériser la faune corallienne d'une région. Et on peut tenir pour certain que la liste est loin d'être close; elle s'allongera encore d'une centaine de formes, au moins, lorsque toute la ceinture corallienne du globe sera aussi bien explorée que l'Indo-Pacifique du Seelark et du Siboga.

Le beau travail de de Man, malheureusement non accompagné des figures si indispensables, m'a obligé à revoir certaines déterminations, en particulier celle du S. neomeris, mais, dans l'ensemble, les espèces communes aux deux régions n'ont pas augmenté de nombre, ou à peu près. J'ai donc pu me borner à reproduire, presque textuellement, mon travail de 1908, en y intercalant les espèces dejà connues, et en y ajoutant de nombreuses figures. Ce mémoire est, en somme, la suite de celui que j'ai publié sur les Alpheidæ des Maldives et Laquedives, aussi ai-je pu être très sobre d'indications bibliographiques. Je n'ai pas non plus reproduit les indications des groupes d'espèces, aujourd'hui classiques. Il serait beaucoup plus intéressant de pouvoir dire pourquoi il faut distinguer tant de formes spécifiques dans cette famille à éthologie si spéciale, mais les raisons que j'en aperçois sont beaucoup trop spéculatives pour que je me risque à les donner ici.

Genre Automate de Man.

1. A. salomoni H. Cout., Bull. Suc. Phil. 1908, p. 2. (Plate 60, fig. 1.)

L'unique spécimen est une ? ovée mesurant $17.5 \mathrm{~mm}$.

Chagos, Salomon Island.

Genre Athanas Leach.

2. A. djiboutensis H. Cout., Alph. M. et L. p. 856, fig. 129.

Chagos, Egmont récif.

Genre Arete Stimpson.

3. A. indicus H. Cout., Alph. M. et L. p. 863, figs. 134-35.

Seychelles, Praslin reef.

4. A. equalis H. Cout., Alph. M. et L. p. 869, fig. 138.

Coetivy, Amirante Bank, 25-80 brasses.

SECOND SERIES-ZOOLOGY, VOL. XVII. 
Genre Alpheopsis H. Cout.

5. A.fissipes H. Cout., Bull. Soc. Phil. 1908, p. 3. (Plate 60, fig. 3.)

L'unique spécimen de l'A. fissipes est une $q$ de $6.5 \mathrm{~mm}$. de longueur, portant 7 oufs seulement, de taille comparable à ceux de l'A. equalis.

Providence, D. 4 (50-78 brasses).

6. A. idiocarpus H. Cout., Bull. Soc. Phil. 1908, p. 6. (Plate 60, fig. 2.)

L'unique spécimen de l'A. idiocarpus est un ô de $7 \mathrm{~mm}$., provenant, comme le précédent, de Providence, $50-78$ brasses.

Ces deux espèces constituent une augmentation de grande importance pour le genre Alpheopsis. Jusqu'à présent, ses deux groupes, equalis à pinces lisses, et trispinosus à pinces sillonnées, paraissent assez faiblement reliés: Peut-être les formes ci-dessus décrites apporteraient-elles précisément le trait d'union cherché, si leurs pinoes de la $I^{\mathbf{e}}$ paire étaient connues.

Genre Synalpheus sp. Bate.

7. S. metaneomeris n. nom. (Plate 60, fig. 4.)

S. neomeris H. Cout., Alpheidæ des Laquedives et Maldives nec de Man. Bull. Soc. Phil. xi. 5, 1908.

S. streptodactylus de Man, Alpheidæ du Siboga, p. 226.

Dans son magnifique travail sur les Alpheidæ recueillis par l'expédition du Siboga, de Man établit définitivement la non-identité du véritable $S$. neomeris de Man avec l'espèce ci-dessus, qui en est évidemment très distincte. Je ne puis toutefois accepter le nom de streptodactylus donné par de Man, le nom correspondant à une variété que je crois toujours valable, et qui est basée sur l'aspect différent des dactyles. Je reproduis ici les 2 figures de mon précédent travail, où l'on voit clairement, mieux que par toute déscription, la différence de forme et de proportion des deux griffes. Cette forme devra donc porter le nom de S. metaneomeris var. streptodactylus.

Quant à $S$. metaneomeris, l'espèce me paraît assez variable. Le sp. ô que j'ai figuré est un de ceux qui ont été communiqués à de Man et lui ont servi à la description de S. streptodactylus. Le scaphocérite s'étend jusqu'au milieu de l'article antennulaire distal, et toutes les épines sont très allongées. Cette différence n'est pas sexuelle autant qu'il en a paru, elle correspond à ce que j’ai appelé la forme "oxyceros" qui marque chez les Synalphées une véritable direction évolutive, si l'on en juge par sa fréquence. Les deux autres spécimens figurés sont des $q$, choisies parmi d'autres exemplaires pour la brièveté des épines antennaires et antennulaires, mais qu'il est impossible par ailleurs de séparer.

Cette espèce est la plus abondamment représentée du genre Synalpheus dans la collection. Elle a été recueillie dans les localités et stations suivantes:

Amirante Bank, st. 1, 2, 3, 5, 6, 9, 11, 13 (20-80 brasses); Saya de Malha, st. 10, 12 (47 et 90 brasses); Cargados Carajos, st. 8, 13, 15, 19, 20 (28--33 brasses); Providence, st. 11 ( 58 brasses).

8. S. gravieri H. Cout., Alph. Mald. et Lacc. p. 870, pl. 70, fig. 2.

Amirante Bank, st. 16, 18, 22 (16-280 brasses); Providence, st. 11 (58 brasses). 
9. S. merospiniger H. Cout., Bull. Soc. Phil. 1908, p. 5. (Plate 60, fig. 5.)

Amirante E. 25 (20-44 brasses). Une q ovée.

10. S. paraneomeris $\mathrm{H}$. Cout.

S. paraneomeris H. Cout., Alph. Mald. et Lacc. p. 872, pl. 71, fig. 7.

Chagos: Salomon, et Coin, Peros. Coetivy.

11. S. paraneomeris, praslini n. var. (Plate 61, fig. 6.)

Quelques spécimens de Praslin récif se montrent nettement distincts par trois caractères : l'angle supéro-interne du basicérite est nettement prolongé en une épine, alors que l'absence d'épine en ce point est une des caractéristiques principales de l'espèce paraneomeris. Le méropodite de la $3^{\mathrm{e}}$ paire est plus large que dans les spécimens typiques, le rapport de ses dimensions étant seulement 3.5 au lieu de $4 \cdot 5$. Enfin les oufs sont notablement plus gros, mesurant $1.3 \mathrm{~mm}$. de grand axe.

Seychelles, Praslin reef, $1 \hat{\delta}, 1$.

12. S. paraneomeris, seychellensis n. var. (Plate 61, fig. 7.)

2 spécimens $q$ provenant des Seychelles différent également du type par quelques caractères nets:

Le basicérite a son angle supéro-interne, sinon épineux, au moins aigu.

Les pattes de la $3^{\mathrm{e}}$ paire ont leur méropodite 4 fois aussi long que large, et le propodite porte 8 épines au lieu de 5 chez les spécimens typiques. C'est à ces exemplaires que j’ai comparés le $S$. otiosus. Par tout ses autres caractères, dimensions du carpocérite, forme du dactyle, telson, cette variété seychellensis ne s'éloigne pas de l'espèce paraneomeris.

Enfin il se trouve parmi des spécimens provenant de Coin, Peros Atoll, un ô de la forme "oxyceros," caractérisé par la longueur de toutes ses épines antennaires et antennulaires; le stylo et le basicérite atteignent sensiblement l'extrémité de l'article antennulaire médian, le scaphocérite, par son épine latérale, dépasse de beaucoup le carpocérite. C'est pour de tels exemplaires que j'ai proposé, sur l'invitation de Miss Rathbun, le nom de paraneomeris prolatus, mais je trouve la dénomination commune "oxyceros" infiniment plus expressive, parce qu'elle indique, comme je l'ai dit plus haut, une direction évolutive très répandue, pouvant servir à expliquer comment se sont constituées de nouvelles espèces.

13. S. otiosus H. Cout., Bull. Soc. Phil. 1908, p. 5. (Plate 61, fig. 8.)

L'espèce se distingue du $S$. paraneomeris $H$. Cout. par les points suivants :

Le carpocérite est seulement 3 fois aussi long que large (au lieu de 4 fois).

Le méropodite de la grande pince est un peu plus épais dans sa moitié proximale, et se termine par une pointe obtuse.

Le méropodite de la $3^{\mathrm{e}}$ paire est seulement 3.5 fois aussi long que large, au lieu de $4 \cdot 5$ fois chez le $S$. paraneomeris, et le propodite qui porte 5 épines est également plus massif.

Le telson est plus large à son extrémité, le rapport de ses bases étant 1.5 au lieu de 1.85 .

Coetivy, un bel exemplaire $\delta$.

14. S. nilandensis H. Cout., Alph. Mald. et Lacc. p. 871, pl. 70, fig. 4.

1 ex. Seychelles, st. 5 (44 brasses). 
15. S. nilandensis oxyceros H. Cout., Alph. Mald. et Lacc. p. 871, pl. 70, fig. 5.

1 ex. Chagos, Egmont, lagoon.

16. S. fossor Paulson. Alph. Mald. et Lacc. p. 872, pl. 70, fig. 6.

1 ex. Chagos, Diego-Garcia, lagoon.

17. S. trimyx H. Cout., Bull. Soc. Phil. 1908, p. 6. (Plate 61, fig. 9.)

L'espèce est très voisine du $S$. fossor Paulson, dont elle se distingue par les points suivants :

Les intervalles sont plus larges entre le rostre et les épines latérales, celles-ci étant, par suite, plus divergentes.

L'écaille du scaphocérite atteint au moins la moitié de l'article antennulaire distal, et l'épine latérale dépasse toujours le carpocérite, qui est 5 fois environ aussi long que large. Chez le $S$. fossor, l'écaille antennaire atteint rarement l'extrémité de l'article antennulaire médian, l'épine latérale est toujours plus courte que le carpocérite, et celui-ci est 6 fois plus long que large.

La grande pince est épineuse chez le $S$. trionyx à son bord palmaire antérieur, et le bord supéro-externe du méropodite se termine également par une forte épine. L'un et l'autre sont inermes chez le $S$. fossor, surtout la paume de la grande pince.

La petite pince du $S$. trionyx a pour proportions: doigts 1 , longueur totale $2 \cdot 4$, hauteur $0 \cdot 8$. Le méropodite, dont le bord supéro-externe se termine en pointe aiguë, est $2 \cdot 1$ à 2.6 fois plus long que large. Il est toujours plus épais que la paume. Chez le $S$. fossor les proportions de la petite pince sont: doigts 1 , longueur totale $2 \cdot 85$, hauteur $1 \cdot 2$, le membre étant plus trapu. Par contre, le méropodite grêle est $2 \cdot 7$ fois aussi long que large, et n'a que 0.6 de la hauteur de la paume.

Le premier segment du carpe est plus long que la somme des 4 autres chez le $S$. trionyx, plus court chez le $S$. fossor.

Les proportions de la $3^{\mathrm{e}}$ paire sont chez le $S$. trionyx: propodite $2 \cdot 35$, carpe 1 , méropodite $2 \cdot 6$, celui-ci $4 \cdot 4$ fois aussi long que large. Chez le $S$. fossor, ces proportions sont: propodite 1.9 environ, carpe 1 , méropodite $2 \cdot 4$, celui-ci 3.55 fois aussi long que large.

Enfin, le dactyle est de forme nettement différente. Chez le S. fossor, la griffe ventrale surnuméraire a ses deux côtés presque perpendiculaires, la griffe dorsale est très accessoire par rapport à la ventrale, et le groupe de ces 2 griffes est séparé du reste du dactyle par une faible dépression dorsale. Chez le $S$. trionyx, la surnuméraire ventrale est aiguë, dirigée en avant; la dorsale est presque aussi longue que la griffe principale, et le dactyle ne montre aucune concavité dorsale.

Saya de Malha, C. 10 (26 brasses), 2 ex. ô et $q$; C. 19 (29 brasses), 1 ex. $q$.

18. S. triunguiculatus Paulson.

Cargados Carajos, st. 8, 13, 17 (30-32 brasses).

19. S. charon Heller.

Chagos : Salomon, Peros, Egmont, récif et lagon. Coetivy.

20. S. biunguiculatus Stimpson. H. Cout., Alph. des Mald. et Lacc. p. 873, pl. 71, fig. 8. Amirante Bank, st. 6, 13, 21 (20-30 brasses). Chagos, Egmont. 
21. S. pachymeris n. nom. (Plate 61, fig. 10.)

S. biunguiculatus var. pachymeris H. Cout., Alph. Mald. et Lacc. p. 873, pl. 71, fig. 9.

Cette forme est nettement distincte du S. biunguiculatus. Elle est représentée dans la collection par quelques spécimens typiques, mais aussi par une forme dont je fais une variété cargadosi et qui s'en distingue par quelques caractères : l'angle supéro-interne du basicérite est à peine épineux, le méropodite de la $3^{\mathrm{e}}$ paire ne porte que 3 épines mobiles sur son bord inférieur, au lieu de 5, entin le propodite n'a que 5 épines au lieu de 8 , et il est plus court, mesurant $1.33 \mathrm{du}$ carpe au lieu de 1.47 chez les spécimens typiques.

Cargados Carajos, 1 sp. + (var. Cargadosi). Providence Island.

22. S. lophodactylus H. Cout., Bull. Soc. Phil. 1908, p. 7. (Plate 61, fig. 11.)

Cette nouvelle espèce se distingue du $S$. biunguiculatıs Stp. par les points suivants:

Le pédoncule antennulaire est plus court, l'article basal ne dépassant guère le rostre, et seulement 4 fois aussi long que large. Le stylocérite ne dépasse pas l'article basal.

L'écaille antennaire atteint l'extrémité de l'article antennulaire médian, et son épine latérale ne le dépasse que de la moitié environ de l'article antennulaire distal.

La basicérite est complètement inerme en dessus, détail qui différencie immédiatement les deux espèces. Le carpocérite dépasse notablement l'antennule, mais il est seulement 4 fois aussi long que large, ayant subi le même raccourcissement que les autres appendices céphaliques.

La grande pince est absente sur le type.

La petite pince a pour proportions: doigts 1 , longueur totale $2 \cdot 73$, hauteur 0.88 . Son méropodite est $3 \cdot 3$ fois aussi long que large. Le doigt mobile porte une brosse de soies sériées sur la moitié distale. Elles sont moins serrées et moins abondantes que dans le groupe lavimanus, mais n'en indiquent pas moins l'étroite parenté qui relie les deux groupes d'espèces. Chez le $S$. biunguiculatus, la petite pince est plus épaisse, et le doigt mobile n'a que des traces insignifiantes de la brosse de soies sériées.

Les pattes de la $3^{\mathrm{e}}$ paire ont pour proportions : propodite $1 \cdot 9$, carpe 1 , méropodite $2 \cdot 43$. Ce dernier, très grêle, est $4 \cdot 5$ fois aussi long que large. Le dactyle est celui du $S$. biunguiculatus.

Lagon de Diego, 1 seul spécimen $q$.

23. S. tumido-manus, Paulson.

S. tumido-manus Paulson. H. Cout., Alph. Mald. et Lacc. p. 876, pl. 73, fig. 14.

Cargados Carajos, st. 2 (30 brasses), 1 ex. §.

24. S. hastilicrassus H. Cout., Alph. Mald. et Lacc. p. 875, pl. 72, fig. 12.

Amirante Bank, st. 21 (30 brasses); Providence, st. 11 (58 brasses).

25. S. sladeni H. Cout., Bull. Soc. Phil. 1908, p. 8. (Plate 62, fig. 12.)

Cette espèce, l'une des plus remarquables recueillies par l'expédition, est jusqu'a présent la seule du groupe lavimanus qui soit présente dans la région indo-pacifique, à l'exception peut-être du S. lavimanus haddoni $\mathrm{H}$. Coutière. Ce groupe est caractérisé, comme je l'ai montré, par la présence constante sur le doigt mobile de la petite pince d'une brosse de longues soies raides disposées en séries transversales, et occupant les $\frac{2}{3}$ de 
l'article. Tout en présentant de façon très nétte ce caractère, la nouvelle espèce se place bien à part dans le groupe lavimanus.

Le bord frontal présente en avant des yeux une avancée considérable, si bien que la pointe du stylocérite est au niveau de celle du rostre, et que l'épine latérale du basicérite n'atteint pas tout à fait les épines latérales du bord frontal.

Le scaphocérite est sensiblement aussi long que l'antennule, et son écaille atteint l'extrémité de l'article antennulaire médian, caractères insolites dans le groupe, ainsi que la faible épine inférieure du basicérite.

Le carpocérite est volumineux, 4.7 fois aussi long que large, plus long que les antennules.

Les fouets des maxillipèdes extemes sont très asymétriques en longueur et surtout en volume. Jignore si cette disposition est fortuite ou constante.

La grande pince a pour proportions: doigts 1, longueur totale $3 \cdot 12$, bauteur 0.84 . Le doigt mobile dépasse un peu le doigt fixe. La paume est cylindrique, et sa grande longueur est également une caractìre insolite.

Par contre, la petite pince ressemble beaucoup à celle du S. longicarpus Herrick, par sa forme, ses proportions, sa brosse de soies sériées.

La $2^{\mathrm{e}}$ paire est grêle, la $3^{\mathrm{e}}$ paire également. Les proportions de cette dernière sont: propodite 2 , carpe 1 , méropodite $2 \cdot 15$, ce dernier article $5 \cdot 4$ fois aussi long que large. La griffe est celle de toutes les espèces lievimanus.

Le telson a pour proportions : largeur distale 1 , largeur proximale $2 \cdot 7$, hanteur 3 environ.

Pour l'avancée du bord frontal, la forme de la grande pince, la gracilité des pattes suivantes, la forme du telson, cette espèce rappelle les espèces du groupe comatularum, jusqu'à présent aussi exclusives à l'Indo-pacifique que le groupe larimanus l'est aux côtes américaines.

Cargados Carajos, B. 2 (30 brasses), 1 seul ex. $\hat{\delta}$.

Genre Alpheus Fabr.

26. A. staphylinus H. Cout., Bull. Soc. Phil. 1908, p. 14. (Plate 62, fig. 13.)

L'espèce est très voisine de l'A. megacheles Hailstone de la Méditerranée et de l'Atlantique. Le rostre est 3 fois aussi long à peu près que les dents sus-orbitaires, il est plus court que l'article antennulaire basal. L'article médian est $\cdot 1 \cdot 5$ fois aussi long que chacun des 2 autres. Le pédonculaire antennulaire, la forte épine latérale du scaphocérite, le carpocérite sont sensiblement égaux.

La grande pince est très semblable à celle de l' $A$. megacheles comme forme et proportions. La petite pince est plus grêle, la paume étant $2 \cdot 43$ fois aussi longue que large, au lieu de 2 fois, le doigt mobile n'est pas élargi, bien qu'il s'agisse d'un $\delta$, les doigts sont légèrement plus courts que la paume.

La $2^{\mathrm{e}}$ paire est très allongée, la somme du carpe et de la pince mesure 0.85 du cephalothorax, et la partie proximale du membre 1.05 de cette même longueur, ce qui distingue immédiatement l'espèce de l'A. megacheles et la rapproche de l'A. hailstonei des Maldives.

La $3^{\mathrm{e}}$ paire mesure $1^{\circ} \mathrm{Z}$ du céphalothorax, alors qu'elle l'égale seulement chez 
l'A. megacheles. Le méropodite est 5 fois aussi long que large, et le propodite est $1 \cdot 4$ fois aussi long que la carpe, aurlieu de $1 \cdot 2$ chez l'A. megacheles.

L'unique spécimen $\hat{\delta}$, long de $12 \mathrm{~mm}$., provient de Salomon Island (Chagos).

27. A. hailstonei H. Cout., Alph. Mald. et Lacc. p. 879, pl. 74, fig. 18.

Seychelles, st. 8, 9 (34 et 37 brasses). Maurice. Saya de Malha, st. 15 (55 brasses).

28. A. paradentipes H. Cout., Alph. Mald. et Lacc. p. 880, pl. 74, fig. 17.

Amirante Bank, st. 25, 26 (20-100 brasses). Providence, st. 11 (58 brasses).

29. A. collumianus Stimpson, Alph. Mald. et Lacc. p. 881.

Amirante Bank, st. 7, 10, 11, 13 (20-85 brasses). Providence, st. 1, 3 (29-39 brasses). Chagos, Salomon. Cargados Carajos, st. 20 (28 brasses).

30. A. seurati H. Cout., Alph. Mald. et Lacc. p. 881, pl. 75, fig. 20.

Amirante Bank, st. 13 (20-25 brasses). Coetivy.

31. A. malhaensis H. Cout., Bull. Soc. Phil. 1908, p. 15. (Plate 62, fig. 14.)

L'espèce se rapproche particulièrement de l'A. seurati $H$. Cout. dont elle se distingue par les points suivants:

Antennes et antennules un peu plus grêles et allongées, y compris l'épine latérale du basicérite.

Bord inférieur de la grande pince entièrement lisse, constriction plus profonde entre la paume et le doigt fixe, extrémité du doigt mobile moins massive.

Carpe de la $2^{\mathrm{e}}$ paire avec les 2 premiers segments presque égaux, le premier légèrement plus long.

Méropodites de la $3^{\mathrm{e}}$ et $4^{\mathrm{e}}$ paires fortement épineux à l'apex inférieur distal, celui de la $3^{\mathrm{e}}$ paire 3.7 fois plus long que large. Proportions du membre : carpe 1 , propodite 1.25 , méropodite 1.9 (au lieu de $1,1 \cdot 45,2$ chez l'A. seurati, où le méropodite est seulement 3 fois aussi long que large, et le propodite également très épais).

Saya de Malha, C. 19 (29 brasses), 1 sp. ㅇ. Amirante, E. 2 (29 brasses), 1 sp. $\delta$.

32. A. macrochirus Richters. H. Cout., Alph. Mald. et Lacc. p. $88 \%$.

Chagos, Salomon Islands. Coetivy.

33. A. ventrosus H. M. Edwards, Alph. Mald. et Lacc. p. 882.

Seychelles, Praslin reef. Chagos: Diego Garcia, barachois; Salomon; Egmont, lagoon. Coetivy.

34. A. gracilis Heller.

Chagos, Salomon. Coetivy.

35. A. gracilis var. alluaudi H. Cout., Alph. Mald. et Lacc. p. 882.

Providence Island, 1 sp. mutilé.

36. A. paragracilis H. Cout., Alph. Mald. et Lacc. p. 883, pl. 76, fig. 22.

Coetivy.

$$
\begin{aligned}
& \text { Alpheus sp.? (Plate 62, tig. 15.) } \\
& \text { Metalpheus n. gen.? }
\end{aligned}
$$

Il s'agit ici d'une forme très remarquable, peut-être identique à l'A. rostratipes Pocock, 
ce que je ne saurais décider en l'absence du type. Cette identité ou valeur spécifique est d'ailleurs secondaires en l'espèce, en présence des différences qui séparent ces formes du genre Alpheus lui-même, et conduisent presque à la formation d'un nouveau genre.

Le rostre émerge entre les échancrures obliques des voutes orbitaires, qu'il égale en longueur. La protection des ophthalmopodes n'est pas assurée du côté antéro-interne.

Comme chez l'A. rostratipes, les articles antennulaires sont aussi larges que longs. Toutefois, le $3^{\mathrm{e}}$ est 1.5 fois aussi long que large. Le stylocérite atteint le milieu de l'article médian. La large écaille du scaphocérite atteint le tiers proximal, et son épine la moitié de l'article distal antennulaire. Le basicérite est volumineux, beaucoup plus haut que long surtout, avec une forte épine latérale, et le carpocérite, dépassant l'antennule de la moitié au moins de l'article distal, n'est guère que 1.5 fois aussi long que large.

Le volume de l'antenne contrastant avec le faible développement du scaphocérite, l'épaisseur des articles et des fouets antennulaires sont des caractères insolites chez Alpheus, rappelant surtout les genres Arete et Alpheopsis.

Le labre possède également un volume excessif. Il descend verticalement entre les bases des antennes, et sa hauteur égale celle du céphalothorax, de façon à constituer une sorte de mufle qui donne à l'espèce une physionomie très spéciale. La partie inférieure en est enfermée, comme dans des valves, entre les psalistomes très élargis des mandibules, dont le palpe est entièrement invisible du dehors. Le psalistome porte une dizaine de faibles dents sur une courte portion de son bord supérieur. Le processus molaire est inclus, comme toujours, entre le labre et les paragnathes, très développés également. C'est là une forme de la mandibule unique jusqu'à présent chez les Alpheidæ.

La maxillule et la maxille ont leur forme normale. Sur le $1^{\mathrm{er}}$ maxillipède, l'article distal du sympodite est largement arrondi, l'endopodite et l'exopodite soudés sur leur tiers proximal, ce dernier avec un très étroit lobe $a$ (Boas).

Le $2^{\mathbf{e}}$ maxillipède a son épipodite en forme de sac membraneux. Le $3^{\mathrm{e}}$ a son article distal foliacé, très élargi (un peu plus de 2 fois aussi long que large) excavé de façon à recevoir la masse du labre, des mandibules et des maxillipèdes précédents, comme chez l'Alpheopsis fissipes H. Cout. L'article distal est conique, pourvu des soies sériées habituelles, sans épines terminales. Il y a un bourgeon d'arthrobranchie à la base du membre, avec une trace de bifurcation.

Par une malchance singulière, les 2 spécimens que j'ai étudiés ne possèdent qu'une seule des pinces de la $1^{\mathrm{e}}$ paire, de même que les types de l'A. rostratipes. Je suis porté à croire que les deux pinces sont semblables. Sur un spécimen sec de l'A. rostratipes (S. Kensington Muséum) la seule pince présente est si volumineuse qu'elle représente bien plutôt la grande. Sur un des deux spécimens de la forme ici étudiée, la pince opposée est en voie de régénération, elle a dépassé le stade de forme indifférente, et apparaît très semblable a son opposée comme aspect général et proportions.

La $2^{\mathrm{e}}$ paire est courte et massive. Les segments du carpe décroissent du $1^{\mathrm{er}}$ au $4^{\mathrm{e}}$, ce dernier plus large que long, le $5^{\mathrm{e}}$ égal au $1^{\mathrm{er}}$. La $3^{\mathrm{e}}$ paire a le méropodite très massif, inerme, 3 fois aussi long que large. Le dactyle est bifide, avec une saillie ventrale surnuméraire plus marquée chez le $\$$.

La $4^{\mathrm{e}}$ paire n'a pas d'épipodite en crochet. Les rames des pléopodes, chez le $\hat{\delta}$, sont 
beaucoup plus courtes que le sympodite, presque dépourvues de soies et de longueur égale. La $2^{\mathrm{e}}$ paire fait exception en ce que la rame interne est étroite et très longue.

Chez la $q$ les rames sont plus longues et aussi plus larges que le sympodite, et le rétinacle de la rame interne est lui-même très élargi, si bien que la rame paraît simplement bifurquée sur son tiers distal.

Volume des fouets antennaires et de l'antenne, incomplète protection des yeux, volume $\mathrm{du}$ labre, forme très spéciale des mandibules, du $2^{\mathrm{e}}$ maxillipède, du $3^{\mathrm{e}}$ maxillipède, des pinces de la $1^{\mathrm{e}}$ paire (?), brièveté de la $2^{\mathrm{e}}$ paire, réduction du nombre des épipodites, forme très spéciale des pléopodes, tels sont les caractères que l'on pourrait invoquer pour la séparation de l'A. rostratipes et des formes affines. Le nouveau genre pourrait recevoir le nom de Metalpheus s'il était conservé.

D'autre part, ces espèces montrent avec l'A. paragracilis $\mathrm{H}$. Cout. une ressemblance très grande, au point que tous leurs caractères différentiels s'y retrouvent, $y$ compris l'absence d'épipodite sur la $4^{\mathrm{e}}$ paire. Mais ces caractères sont pour la plupart atténués. Il en est ainsi pour le volume de l'antenne et sa disproportion d'avec le scaphocérite, pour le volume du labre, la grandeur du psalistome des mandibules, du sympodite du $l^{\mathrm{er}}$ maxillipède, la largeur de l'article basal du $3^{\mathrm{e}}$ maxillipède, le volume de la petite pince, la brièveté de la $2^{\mathrm{e}}$ paire, pour la forme même des pléopodes. En un mot, l'A. paragracilis ne possède plus en propre qu'un seul caractère le séparant nettement du genre Alpheus, l'épipodite du $4^{\mathrm{e}}$ péréiopode. Et si l'on passe à des espèces telles que l'A. socialis, également très voisine, ce dernier détail disparaît.

On voit donc l'intérêt qui s'attache à la connaissance plus complète de l'A. rostratipes (et de l'A. sp ? qui en est peut-être distinct) surtout pour savoir si la grande pince est ou non celle d'un Alpheus.

On peut noter que l'Alpheopsis fissipes, la nouvelle espèce décrite plus avant, possède, seule du genre, les maxillipèdes de la $3^{\mathrm{e}}$ paire très élargis et les dactyles bifides. Si, là encore, les pinces de la $1^{\text {re }}$ paire étaient connues, peut-être possèderait-on un repère précieux pour fixer la validité du genre Metalpheus. Par son rostre, l'Alpheopsis fissipes se rattache à l'A. equalis, dont les pinces ne sont pas sillonnées. On conçoit très bien dès lors qu'une forme telle que Metalpheus, dans l'hypothèse où ses pinces seraient semblables et non sillonnées, soit sortie d'une autre telle que l'Alpheopsis fissipes.

D'autre part, le groupe megacheles, parmi les Alphées, présente comme je l'ai montré, les ressemblances les plus étroites avec les Alpheopsis tel que l'A. trispinosus et l'A. chilensis, à pinces sillonnées. Comme les 2 sections du genre Alpheopsis sont fort voisines, il n'est pas étonnant de rencontrer chez leurs dérivés Alpheus et Metalpheus des convergences comme celles qui rapprochent l'A. rostratipes et l'A. paragracilis.

\section{Alpheus amirantei H. Cout., Bull. Soc. Phil. 1908, p. 15. (Plate 63, fig. 16.)}

Cette espèce se rapproche plus de l' $A$. paragracilis $\mathrm{H}$. Cout. que d'aucune autre, mais elle en est facilement séparable.

Le rostre étroit se continue par une faible crête, un peu élargie derrière les voûtes orbitaires ; il est séparé de celles-ci, en avant, par deux très faibles sinus concaves du bord frontal, le reste du bord étant régulièrement convexe et inerme. 
Le stylocérite est plus court que l'article antennulaire basal, ou à peine aussi long, le basicérite de l'antenne est à peu près inerme.

Le bord inférieur de la grande pince est droit, sans trace de constriction. Le bord supérieur porte au contraire une constriction transverse el un sillon longitudinal du côté inféro-interne. Du côte supéro-externe est un second sillon longitudinal très faible. Le méropodite porte une forte épine à son bord inférieur interne.

La petite pince diffère de celle de l'A. paragracilis par sa taille relative moindre, les doigts égaux à la paume, le méropodite pourvu d'une forte épine.

Sur la $2^{\mathrm{e}}$ paire, le $1^{\mathrm{er}}$ segment du carpe n'a guère que le tiers du second.

La $3^{\mathrm{e}}$ paire et la $4^{\mathrm{e}}$ ont le méropodite fortement épineux à son bord inférieur distal, le propodite est seulement un peu plus long que le carpe, l'un et l'autre sont beaucoup plus faibles que le méropodite. Le dactyle est simple. C'est une forme du membre surtout fréquente dans le groupe crinitus.

Amirante E. 11 (25-80 brasses), 2 spécimens 8.

38. Alpheus baculifer H. Cout., Bull. Soc. Phil. 1908, p. 16. (Plate 63, fig. 17.)

Je place ici cette très remarquable espèce. Elle représente un cas extrême d'allongement des pinces chez une forme qui, par la plupart de ses caractères, rentre dans le groupe obeso-manus. C'est le parallèle de l'A. cylindricus Kingsley pour le groupe megacheles.

Le rostre, très court, dépasse à peine les voûtes orbitaires très saillantes, et se continue en arrière par une crête assez marquée. L'article antennulaire médian est 1.5 fois aussi long que chacun des deux autres, le stylocérite est plus court que l'article médian et ne fait qu'une faible saillie latérale. Le scaphocérite ne possède qu'une écaille rudimentaire, et son épine latérale ne dépasse pas le tiers distal de l'article antennulaire médian. Le carpocérite est à peine plus long que ce même article.

Les pattes de la $1^{\mathrm{e}}$ paire sont extrêmement caractéristiques. La grande pince a la paume cylindrique, 5.5 fois aussi longue que large. Elle est tronquée à l'extrémité ; le doigt mobile, en "porte à faux," sur plus de la moitié de sa longueur, est dirigé presque perpendiculairement à la paume. Il n'y a plus trace de sillons palmaires. Le méropodite est également très allongé.

La paume de la petite pince est de même forme, mais les doigts sont parallèles, égaux, et dans le prolongement du membre. Ses proportions sont: doigts 1 , longueur totale $4 \cdot 34$, hauteur 0.55 .

Les pattes de la $2^{\mathrm{e}}$ paire sont très inégales, comme dans la plupart des espèces obesomanus. La plus longue mesure 1.66 fois celle de la $3^{\mathrm{e}}$ paire, la plus petite 1.33 fois. Le second segment du carpe est très légèrement plus long que le premier, la pince distale a les doigts très courts.

La $3^{\mathrm{e}}$ paire a pour proportions : carpe 1 , propodite $1 \cdot 07$, méropodite $1 \cdot 46$. Ce dernier est inerme, et $3 \cdot 6$ fois aussi long que large.

L'unique spécimen est une $q$ venant de muer dont les pleurons abdominaux sont très grands, comme il est de règle dans ce groupe d'espèces. Le telson et les uropodes sont dans le même cas ; la rame interne de ces derniers a son bord externe triangulaire, la suture de la rame externe porte 2 courtes épines.

Ile du Coin, Peros (Chagos), une $q$ de $25 \mathrm{~mm}$. 
39. A. ovaliceps H. Cout.; Alph. Mald. et Lacc. p. 888, pl. 77, fig. 27.

Chagos : Coin, Peros.

40. A. stanleyi H. Cout., Bull. Soc. Phil. 1908, p. 17. (Plate 63, fig. 18.)

Cette espèce se rapproche beaucoup de l'A. ascensionis Ortmann, de l'A. architectus de Man, de l'A. styliceps $\mathrm{H}$. Coutière.

La forme du bord frontal est très semblable à celle de l'A. styliceps, les voutes orbitaires étant toutefois reportées plus en arrière encore. L'écaille du scaphocérite atteint le tiers distal de l'article antennulaire médian, tandis qu'en revanche l'épine latérale atteint à peine le milieu de l'article distal. Le carpocérite ne dépasse que très faiblement l'antennule.

La grande pince est celle de l'A. ovaliceps $\mathrm{H}$. Coutière, et se distingue par suite de celle des 3 espèces précitées. Le doigt mobile est toutefois beaucoup plus en "porte à faux." Les sillons palmaires sont à peu près nuls. Ses proportions sont : doigt mobile 1, longueur totale $3 \cdot 4$, hauteur $1 \cdot 4$.

La petite pince a pour proportions : doigts 1, longueur totale 2, hauteur 172. Elle est, par suite, de forme banale, et bien distincte de celle de l'A. styliceps. Les doigts sont béants, l'inférieur très large ; leur bord inféro-externe excavé porte une rangée de fortes soies courtes et espacées, au nombre d'une dizaine.

Sur la $2^{\mathrm{e}}$ paire, le $2^{\mathrm{e}}$ segment du carpe est un peu plus court que le premier.

La $3^{\mathrm{e}}$ paire a pour proportions : propodite $1 \cdot 4$, carpe 1 , méropodite $2 \cdot 25$. Ce dernier, presque 5 fois aussi long que large, porte une forte épine à son angle inférieur distal. Il en est de même sur la $4^{\mathrm{e}}$ paire. Le dactyle est très court, sans trace de bifurcation.

Le telson a pour proportions : petite base 1, grande base 2 , hauteur $3 \cdot 27$. Le bord de l'uropode interne porte une série de courtes épines.

Chez l' $A$. architectus et l'A. ascensionis, indépendamment des autres différences, les méropodites 3 et 4 sont inermes.

Amirante E. 21 (30 brasses), un unique sp. ô de $18 \mathrm{~mm}$.

41. A. microstylus H. Cout., Alph. Mald. et Lacc. p. 884, pl. 76, fig. 23. Coetivy.

42. A. phrygianus H. Cout., Alph. Mald. et Lacc. p. 886, pl. 77, fig. 25.

Chagos: Salomon Island, lagoon. Providence, st. 1 (39 brasses). Amirante, st. 21 (30 brasses).

43. A. lutini H. Cout., Alph. Mald. et Lacc. p. 885, pl. 76, fig. 24. Coetivy.

44. A. bradypus H. Cout., Alph. Mald. et Lace. p. 891, pls. 78-79, fig. 30. Coetivy.

45. A. bucephalus H. Cout., Alph. Mald. et Lacc. p. 890, pl. 78, fig. 29.

Providence, st. 4, 7 (50 et 70 brasses). Chagos: Salomon; Diego Garcia, barachois. Amirante Bank, st. 9, 13, 16, 29 (20-44 brasses). Coetivy.

46. A. aculeipes H. Cout., Alph. Mald. et Lacc. p. 892, pl. 79, fig. 31.

Amirante Bank, st. 2, 9, 11, 13, 16, 21 (20-39 brasses). Seychelles, st. 1, 5, 8, 9 (20-44 brasses). Saya de Malha, st. 10,19 (90 et 55 brasses). Coetivy, récif. Chagos: Diego Garcia.

47. A. paraculeipes H. Cout., Alph. Mald. et Lacc. p. 894, pls. 79-80, fig. 32 .

Amirante Bank, st. 13, 21 (20-30 brasses). 
48. A. providencei H. Cout., Bull. Soc. Phil. 1908, p. 18. (Plate 63, fig. 19.)

Le difficile groupe crinitus, déjà si riche en espèces, a fourni encore ici plusieurs formes nouvelles, séparées des formes connues par de minimes différences.

Par la forme du bord frontal et des appendices céphaliques, 1'A. providencei rappelle absolument l' $A$. aculeipes $H$. Coutière. Ici, toutefois, le rostre fait à peine saillie en avant; sa crête médiane est au contraire plus forte, et s'élargit plus rapidement derrière les voutes orbitaires.

Par ses autres caractères, l'espèce se rapproche davantage de l'A. paraculeipes $\mathrm{H}$. Coutière, sans être toutefois intermédiaire entre les deux formes précitées.

La grande pince, dont les proportions sont: doigts 1, longueur totale 3.33, hauteur 1.5 , a une forme régulièrement ovoïde; ses doigts sont fortement infléchis en dedans. Le méropodite épineux est $2 \% 2$ fois plus long que large.

La petite pince $(q)$ est très. semblable à celle de l'A. paraculeipes. Les doigts sont seulement plus longs, ses proportions étant: doigts 1 , longueur totale $2 \cdot 18$, hauteur 0.8 .

Les deux premiers segments du carpe sont entre eux comme 1 et 1.95 , rapport moindre que dans les deux espèces précitées.

Pour la $3^{\mathrm{e}}$ paire, les proportions sont: propodite $1 \cdot 5$, carpe 1 , méropodite $2 \cdot 3$. Ce dernier 4 fois plus long que large. L'épine mérale est dans le prolongement du bord inférieur, étroite et très aiguë. Le bord inférieur interne est absolument nu, d'où une différence notable d'avec les $A$, aculeipes et paraculeipes.

Le bord externe du carpe porte une épine et 8-10 soies, mais celles-ci sont effilées, molles, et bien différentes de celles de l'A. paraculeipes. Le propodite n'a pas d'épine distale au bord supérieur. Le dactyle porte au bord ventral ure saillie surnuméraire très nette. L'ischiopodite n'a pas d'épine enfoncée. Le méropodite de la $4^{\mathrm{e}}$ paire n'est pas épineux.

L'espèce est encore plus voisine de l'A. alcyone de Man. Dans cette dernière forme, le scaphocérite a son bord externe concave, son épine latérale plus longue; le méropodite de la petite pince est inerme; le premier segment du carpe mesure seulement le tiers du second, et il est surtout plus petit que le $5^{\mathrm{e}}$. Le méropodite de la $3^{\mathrm{e}}$ paire porte quelques spinules courbées en $\mathrm{S}$, le dactyle n'est pas bifide; le carpe de la $4^{\mathrm{e}}$ paire est semblable à celui de la $3^{\text {e }}$. Enfin, les œufs sont rares et volumineux, donnant vraisemblablement naissance à des larves mysis.

On peut considérer l'A. alcyone et l'A. providencei comme deux formes pœcilogoniques, mais, ici comme dans la grande majorité des cas connus, il s'ajoute au caractère tiré du volume des ceufs une série de menues différences montrant que les deux formes sont bien spécifiquement distinctes.

Providence D. 4 ( $50-78$ brasses), 1 sp. ․

49. A. alcyone de Man? H. Cout., Alph. Mald. et Lacc. p. 896.

Amirante Bank, st. 13 (20-25 brasses).

50. A. paralcyone H. Cout., Alph. Mald. et Lacc. p. 895, pls. $80-81$, fig. 34 .

Amirante Bank, st. 11, 13, 21 (20-80 brasses). Seychelles, st. 8 (34 brasses). 
51. A. superciliaris H. Cout., Alph. Mald. et Lacc. p. 896, pl. 81, fig. 35 .

Amirante Bank, st. 21, 25 (209-160 et 44-20 brasses).

52. A. pachychirus Stimpson.

Chagos: Salomon; Egmont. Coetivy.

Cowles a minutieusement décrit, récemment, la façon dont cette espèce se construisait un tube à l'aide d'Algues vertes pour s'y loger par couples. Il s'agit plus probablement de l'A. frontalis $=$ Betaus utricola Richters.

53. A. frontalis H. M. Edwards.

Amirante Bank, st. 9, 11, 13 (20-80 brasses). Chagos: Egmont ; Salomon. Seychelles: st. 2 (31 brasses); Praslin, récif. Coetivy.

54. A. clypeatus H. Cout., Alph. Mald. et Lacc. p. 897, pls. 81-82, fig. 36.

Chagos: Egmont, récif.

55. A. adamastor H. Cout., Bull. Soc. Phil. 1908, p. 19. (Plate 64, fig. 20.)

Cette espèce est extrêmement voisine de l'A. clypeatus H. Coutière. Elle en diffère par les points suivants:

Le bord frontal présente une avancée convexe en deçà de chaque voâte orbitaire, mais, au lieu de se réunir en un rostre saillant, les voutes sont séparées par un sinus médian, aussi large que chacune d'elles. La crête mousse du rostre, qui paraît s'arrêter au bord de ce sinus, se continue en réalité un peu en avant et sur un plan inférieur, en une pointe étroite et très courte.

Le basicérite porte une épine beaucoup plus faible que chez l'A. clypeatus.

La grande pince a pour proportions: doigts 1, longueur totale 3, hauteur 1:34. Elle est done légèrement plus massive que chez l'A. clypeatus, où les doigts ont aussi $1 / 3$ de la longueur totale, mais où le second rapport est 1.23 seulement.

La petite pince présente des différences bien plus notables. Elle a pour proportions : doigts 1 , longueur totale $2 \cdot 36$, hauteur $0 \cdot 75$. Elle est donc 3 fois plus longue que haute, au lieu de $2 \cdot 6$ chez l' $A$. clypeatus $\delta$. Elle rappelle par suite les proportions de l'appendice chez la $q$ de l'espèce précitée, mais le doigt mobile est ici notablement élargi. Il est vraisemblable que la différence sexuelle dans la forme de la petite pince, chez l'A. adamastor. dont la $q$ est inconnue, porte sur ce détail du doigt mobile, et qu'elle est par suite assez faible.

Le méropodite inerme est à peine plus court que la pince et presque 3 fois aussi long

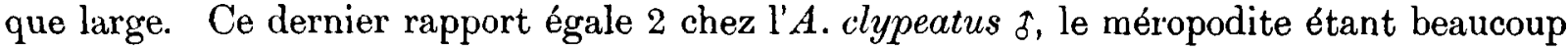
plus court que la pince.

Sur la $2^{\mathrm{e}}$ paire, le $1^{\mathrm{er}}$ segment du carpe égale $1^{\cdot} \mathrm{C}$ fois le second. Ils sont sensiblement égaux chez l'A. clypeatus.

La $3^{\mathrm{e}}$ paire est très semblable comme proportions et forme à celle de l'A. clypeatus, mais le méropodite est 4 fois aussi long que large, au lieu de 3.5 fois.

La forme du bord frontal et les proportions de la petite pince du ô permettent aussi de distinguer aisément cette espèce de l'A. pachychirus Stimpson.

Salomon (Chagos), un unique spécimen $\delta$. 
56. A. longecarinatus Hilgendorf.

Amirante Bank, E. 13 (20-25 brasses). Providence, D. 1 (39 brasses).

57. A. insignis Heller.

Chagos: Egmont; Salomon. Coetivy.

58. A. larceloti H. Cout., Alph. Mald. et Lacc. p. 901, pl. 83, fig. 39.

Amirante Bank, E. 11 (25-80 brasses).

59. A. splendidus H. Cout., Bull. Mus. Paris, 1897, no. 6, p. 235.

Seychelles : Praslin, récif.

60. A. dasycheles H. Cout., Bull. Soc. Phil. 1908, p. 21. (Plate 64, fig. 21.)

L'espèce se rapproche de l'A. gracilipes Stimpson par la forme du rostre. C'est une large pointe triangulaire a bords légèrement concaves, 1.5 fois aussi longue que large à la base. Il y a sur la ligne médiane une trace de l'épine gastrique. Les bords du rostre surplombent les sillons rostro-orbitaires comme chez l'A. gracilipes, et les voûtes orbitaires présentent en avant une saillie obtuse, ne dépassant pas le bord frontal sinueux. La pointe du rostre n'atteint pas l'extrémité de l'article antennulaire basal, que le stylocérite dépasse légèrement.

L'écaille du scaphocérite égale le pédoncule antennulaire, que son épine latérale dépasse des $3 / 4$ environ de l'article distal. L'épine latérale du basicérite atteint en avant aussi loin que le rostre, le carpocérite ne dépasse pas l'extrémité de l'article antennulaire médian.

Les pinces de la $1^{\mathrm{e}}$ paire sont cylindriques et entières, sans traces de sillons et de lobes. Elles possèdent un revêtement assez dense de longues soies sur leur face inféro-externe. La plus grande a pour proportions: doigts 1 , longueur totale $3 \cdot 2$, hauteur $0 \cdot 9$.

La plus petite: doigts 1 , longueur totale $2 \cdot 38$, hauteur 0.5 . Sur la $2^{\mathrm{e}}$ paire, le $1^{\mathrm{er}}$ segment égale 1.2 fois le second. La $3^{\mathrm{e}}$ paire a pour proportions : propodite 1.64 , carpe 1 , méropodite 1:88. Ce dernier est 5 fois aussi long que large, l'ensemble du membre étant grêle, mais le méropodite porte une forte épine à son apex inférieur. Le carpe se termine également par une épine. Le dactyle est simple. Il y a une épine enfoncée mobile sur l'ischiopodite.

Seychelles, F. 9 (37 brasses), 2 sp. $\delta$ et $q$, le plus grand mesurant $22 \mathrm{~mm}$.

61. A. percyi H. Cout., Bull. Soc. Phil. 1908, p. 21. (Plate 64, fig. 22.)

Cette espèce se rapproche également de l'A. gracilipes, plus même que la précédente.

Le rostre a la même forme lancéolée, à bords surplomblant les sillons rostro-orbitaires. Mais les vouttes orbitaires sont régulièrement hémisphériques, sans lobe antérieur saillant, de sorte qu'entre elles, le rostre et le bord frontal, il n'existe pas la partie plane du sillon rostro-orbitaire brusquement élargi en avant que l'on remarque chez l'A. gracilipes.

Le rostre atteint, du même que le stylocérite, la longueur de l'article antennulaire basal.

L'écaille antennaire atteint au moins l'extrémité du pédoncule antennulaire, qu'elle dépasse même notablement chez les grands spécimens. Son épine latérale et le carpocérite comme dans l'espèce précédente, et comme chez l'A. gracilipes.

Les pattes de la $1^{\mathrm{e}}$ paire ne diffèrent que très peu de celles de l'A. gracilipes. La grande pince est entaillée d'un profond sillon transverse un peu avant l'articulation du doigt mobile; la petite pince, “balaniceps" chez les $\hat{\delta}$, est munie d'une forte épine sur 
chacun des condyles articulaires du doigt mobile. Les bords supéro-externe et inféroexterne du méropodite se terminent chacun par une forte épine, sur les 2 pinces.

Sur la $2^{\mathrm{e}}$ paire, les 2 premiers segments du carpe, presque égaux, sont entre eux comme 1, 1-15. J'ai étudié des spécimens jeunes où le rapport était sensiblement inverse.

La $3^{\mathrm{e}}$ paire est très semblable à celle de l'espèce précédente comme forme et proportions.

Cargados Carajos (30 brasses), $2 \mathrm{sp}$. $\hat{\jmath}$ et $q$, le plus grand mesurant $40 \mathrm{~mm}$. Amirante E. 11 (25-50 brasses), 1 petit sp. + . Coetivy, 1 petit sp. $\delta$.

62. A. coetivensis H. Cout., Bull. Soc. Phil. 1908, p. 20. (Plate 64, fig. 23.)

L'espèce se rapproche beaucoup de l'A. paracrinitus Miers et de la forme que j'ai décrite comme var. bengalensis de cette dernière.

Les voates orbitaires sont ici atténuées en forme d'épines plates mesurant à peu près la moitié de l'article antennulaire médian. Le scaphocérite a son écaille plus large, son épine latérale moins grande que dans les deux formes précitées, et le carpocérite est plus volumineux.

La grande et la petite pince sont tout à fait celles de l'A. paracrinitus benyalensis comme forme et proportions, la petite pince étant inerme sur le condyle articulaire externe du doigt mobile. Les méropodites sont inermes.

Sur la $2^{\mathrm{e}}$ paire, le premier segment du carpe est $1 \cdot 7$ fois aussi long que le $2^{\mathrm{e}}$, sensiblement comme chez l'A. paracrinitus.

Sur la $3^{\mathrm{e}}$ paire, très grêle, les proportions sont: propodite $1 \cdot 45$, carpe 1 , méropodite $1 \cdot 7$, le membre étant encore plus allongé que chez l'A. paracrinitus.

Coetivy, $1 \mathrm{sp} .8$. Chagos: Salomon, $1 \mathrm{sp}$. $q$ avec sa petite pince seule présente.

63. A. alpheopsides H. Cout., Alph. Mald. et Lacc. p. 901, pl. 83, fig. 40. Chagos: Salomon.

64. A. paralpheopsides H. Cout. Chagos: Salomon.

65. A. parvirostris Dana. Chagos: Egmont; Salomon. Seychelles : Praslin. Coetivy.

66. A. hippothoë de Man. Amirante Bank, E. 13 (20-25 brasses).

67. A. bouvieri H. M. Edwards, Alph. Mald. et Lacc. p. 907, pl. 85, fig. 44. Chagos: Salomon; Coin, Peros. Cargados Carajos. Coetivy.

68. A. bastardi H. Cout., Alph. Mald. et Lacc. p. 907, pl. 87, fig. 45. Chagos: Salomon; Coin, Peros. Coetivy.

69. A. pacificus Dana, Alph. Mald. et Lacc. p. 909, pls. 85-86, fig. 47. Chagos: Salomon; Coin, Peros.

70. A. audouini H. Cout., Alph. Mald. et Lacc. p. 911, pl. 87, fig. 52. Chagos: Salomon.

71. A. strenuus Dana, Alph. Mald. et Lacc. p. 913, pl. 87, fig. 53. Chagos: Salomon.

72. A. strenuus angulatus H. Cout., Alph. Mald. et Lacc. p.914. (Chagos: Salomon. Amirante Bank, E. 11 (25-80 brasses).

73. A. leptochims H. Cout., Alph. Mald. et Lacc. p. 914, pl. 87, fig. 54. Chagos: Egmont; Salomon. Amirante Bank, E. 11 (25-80 brasses). Cargadas Carajos. 


\section{EXPLICATION DES PLANCHES 60-64.}

Fig. I. A utomate salomoni $\mathrm{H}$. Cout., région céphalique vue en dessus; $\mathbf{1}^{\prime}$, région céphalique vue latéralement; $1 a$, patte; $1 b$, telson et uropodes.

Fig. 2. Alpheopsis idiocarpus H. Cout., région céphalique vue en dessus; $2 a$, patte de la deuxième paire; $2 b$, patte; $2 c$, telson et uropodes.

Fig. 3. Alpheopsis fissipes H. Cout., région céphalique vue en dessus; $3 a$, maxillipède III ; $3 b, 2^{e}$ péréiopode; $3 c$, patte; $3 c^{\prime}$, dactylopodite; $3 d$, œuf.

Fig. 4. Synalpheus metaneomeris n. nom., région céphalique vue en dessus $\left(\sigma^{\prime}\right) ; 4^{\prime}, 4^{\prime \prime}$, la même $(q)$; $4 a$, détail de l'antenne $4, b, 3^{\mathrm{e}}$ patte $; 4 c, 4^{\mathrm{e}}$ patte $; 4 c^{\prime}$, dactylopodite ; $4 c^{\prime \prime}$, dactylopodite ; $4 c^{\prime \prime \prime}$, var. streptodactylus dactylopodite; $4 d$, telson; $4 e$, œuf.

Fig. 5. Synalpheus merospiniger $H$. Cout., région céphalique vue en dessus; $5 a$, détails de l'antenne; $5 b$, patte; $5 c$, dactylopodite; $5 d$, telson; 5 e, œuf.

Fig. 6. Synulpheus paraneomeris praslini n. var., région céphalique vue en dessus; $6 a$, patte ; $6 b$, ceuf.

Fig. 7. Synalpheus paraneomeris seychellensis n. var., région céphalique vue en dessus; 7 a, dactylopodite.

Fig. 8. Synalpheus otiosus H. Cout., région céphalique vue en dessus; $8 a$, détails de l'antenne; $8 b$, grande pince; $8 b^{\prime}$, la même, méropodite ct carpe; $8 c$, petite pince ; $8 c^{\prime}$, la mêtme, méropodite et carpe; $8 d$, patte ; $8 d^{\prime}$, dactylopodite; $8 f$, telson.

Fig. 9. Synalpheus trionyx $\mathrm{H}$. Cout., région céphalique vue en dessus; $9 a$, détails de l'antenne; $9 b$, grande pince; $9 b^{\prime}$, - méropodite et carpe $: 9 c$, petite pince; $9 d, 2^{\mathfrak{c}}$ péréiopode; $9 f$, patte; $9 f^{\prime}$, dactylopodite.

Fig. 10. Synalpheus pachymeris cargadosi n. var., région céphalique vue en dessus; $10 a$, patte.

Fig. 11. Synalpheus lophodactylus H. Cout., région céphalique vue en dessus; 11 a, détails de لlantenne; $11 b$, petite pince; $11 c, 2^{\bullet}$ péréiopode; $11 d$, patte; $11 d^{\prime}$, dactylopodite; $11 f$, telson.

Fig. 12. Synalpheus sladeni $\mathrm{H}$ : Cout., région céphalique vue en dessus; $12 a$, grande pince; $12 b$, petite pince; $12 c, 2^{\circ}$ péréiopode; $12 d$, patte; $12 d^{\prime}$, dactylopodite; $12 f$, telson.

Fig. 13. Alpheus staphylinus $\mathrm{H}$. Cout., région céphalique vue en dessus; $13^{\prime}$, région céphalique vue latéralement; $13 a$, grande pince; $13 a^{\prime}$, petite pince; $13 a^{\prime \prime}$, la même, méropodite; $13 b, 2^{e}$ péréiopode; $13 c$, patté.

Fig. 14. Alpheus malhaensis H. Cout., région céphalique vue en dessus; $14 a$, grande pince; $14 a^{\prime}$, la même, méropodite et carpe; $14 b, 2^{e}$ péréiopode; $14 c$, patte.

Fig. 15. Alpheus sp., région céphalique vue en dessus; 15', région céphalique vue latéralement; $15 a$, mandibule; $15 \mathrm{~b}$, maxillipède $\mathrm{I} ; 15 \mathrm{c}$, maxillipède $I I I ; 15 d$, grande pince: $15 \mathrm{f}, 2^{\mathrm{e}}$ péréiopode; $15 g$, patte ; $15 g^{\prime}$, dactylopudite; $15 h$, pléopodes de la fémelle; $15 h^{\prime}$, pléopodes du male.

Fig. 16. Alpheus amirantei H. Cout., région céphalique vue en dessus; $16 a$, grande pince; $16 a^{\prime}$, la même, méropodite et carpe; $16 b$; petite pince; $16 c, 2^{\mathrm{c}}$ péréiopode; $16 d$, patte.

Fig. 17. Alpheus baculifer $\mathrm{H}$. Cout., région céphalique vue en dessus; 17 a, grande pince; $17 b$, petite pince; $17 c, 2^{\mathrm{c}}$ péréiopode; $17 d$, patte; $17 f$, telson et uropodes.

Fig. 18. Alpheus stanleyi $\mathrm{H}$. Cout., région céphalique vue en dessus; $18^{\prime}$, région céphàlique vue latéralement; $18 a$, détail de maxillipède $111 ; 18 b$, grande pince ; $18 b^{\prime}$, la même, méropodite et carpe ; $18 c$, petite pince; $18 c^{\prime}$, détails de la petite pince; $18 d, 2^{\mathrm{c}}$ péréiopode; $18 f$, telson et uropodes.

Fig. 19. Alpheus providencei H. Cout., région céphalique vue en dessus; $19 u$, grande pince; 19 a', la même, méropodite et carpe; $19 \mathrm{~b}$, petite pince; $19 \mathrm{c}, 2^{\circ}$ péréiopode; $19 \mathrm{~d}$, patte; $19 \mathrm{~d}^{\prime}$, dactylopodite ; $19 \mathrm{f}$, détails; $19 \mathrm{~g}$, telson.

Fig. 20. Alpheus adanıastor $\mathrm{H}$. Cout., région céphalique vue en dessus; $20 a$, détail de maxillipède $111 ; 20 b$, grande pince; $20 c$, petite pince; $20 d, 2^{\mathrm{c}}$ péréiopode ; $20 f$, patte.

Fig. 21. Alpheus dasycheles $\mathrm{H}$. Cout., région céphalique vue en dessus; $21 a$, grande pince; $21 b$, petite pince; $21 c, 2^{\circ}$ péréiopode; $21 d$, patte; $21 f$, telson et uropodes.

Fig. 22. Alpheus percyi $\mathrm{H}$. Cout., région céphalique vue en dessus; 22 ', région céphalique vue latéralement; $22 a$, grande pince: $22 b$, petite pince; $22 c$, $2^{\mathrm{e}}$ péréjopode; $22 d$, patte; $22 f$, telson et uropodes.

Fig. 23. Alpheus coetivensis $\mathrm{H}$. Cout., région céphalique vue en dessus; $23 a$, grande pince; $23 b$, petite pince; $23 b^{\prime}$, la même, méropodite et carpe; $23 c, 2^{\mathrm{e}}$ péréiopode; $233 d$, patte. 

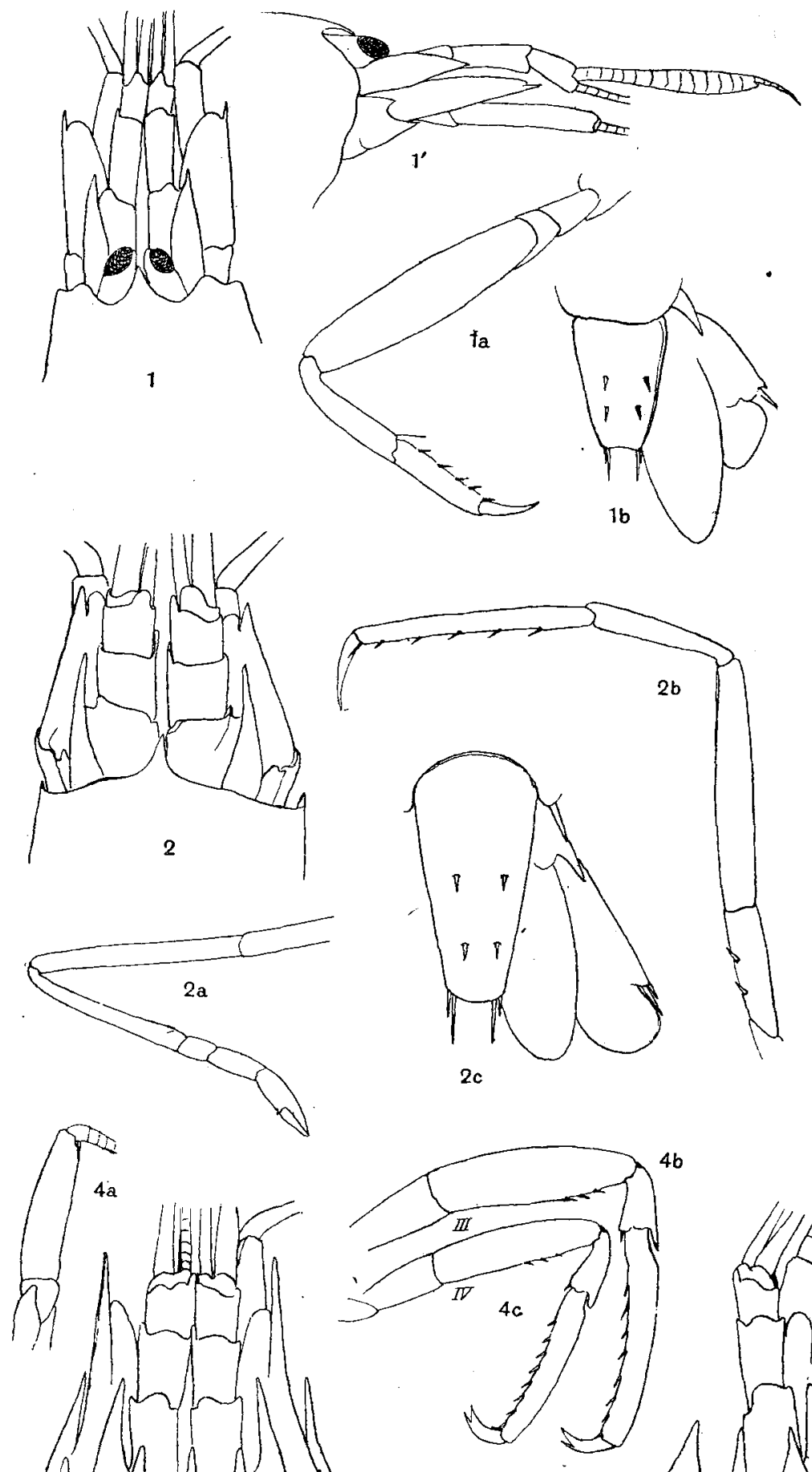

$2 c$

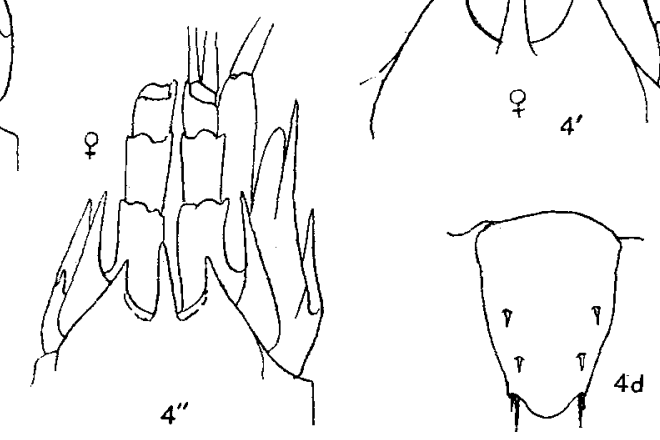

ALPHEIDAE
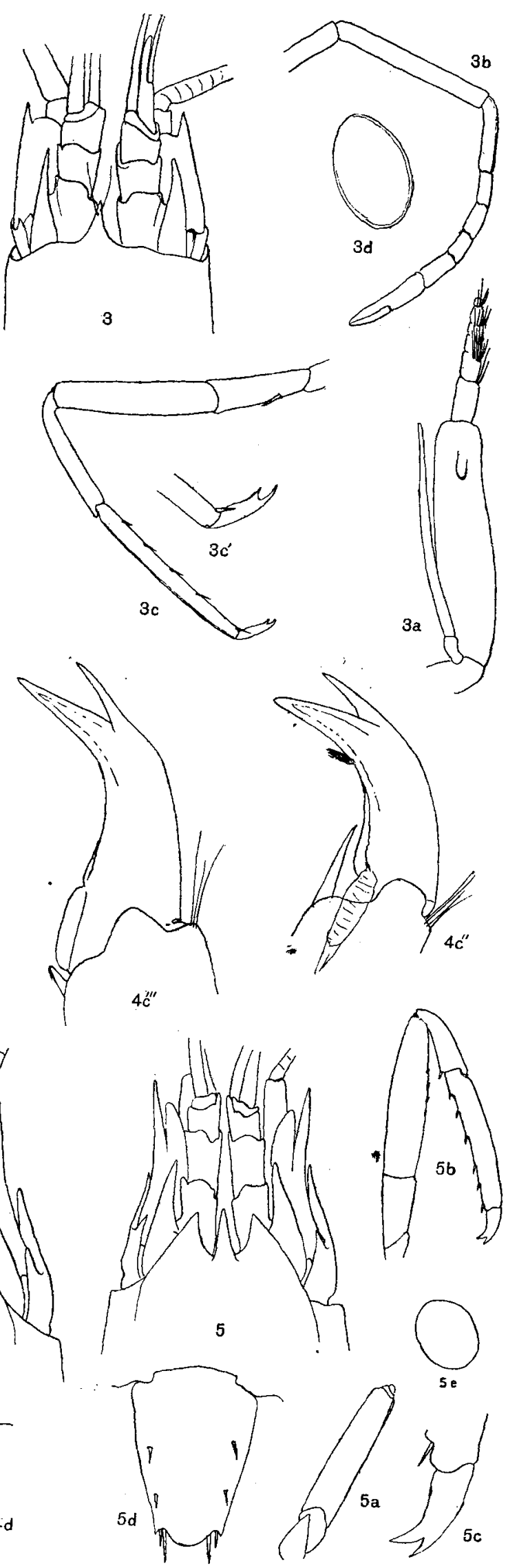

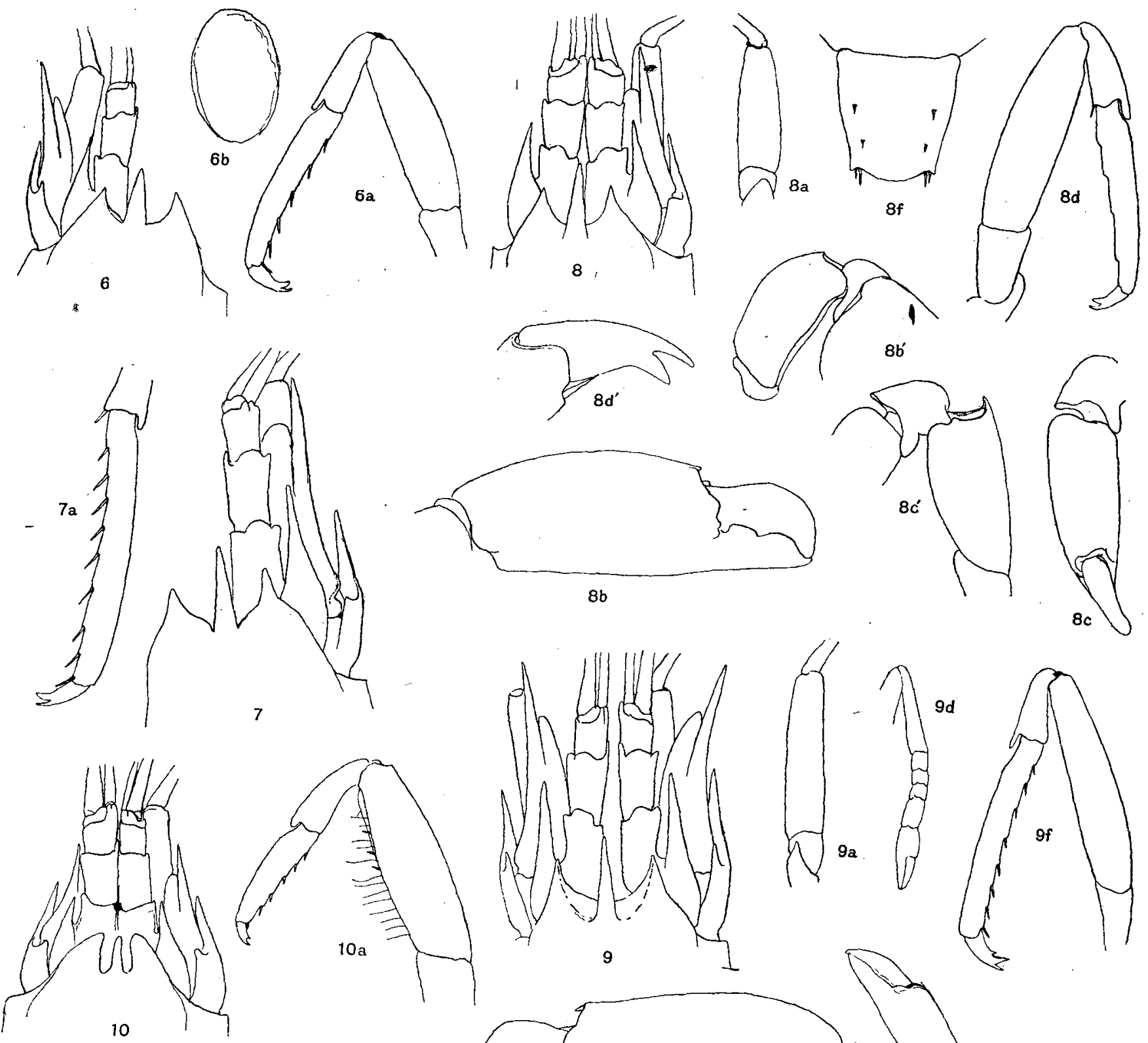

$8 b$
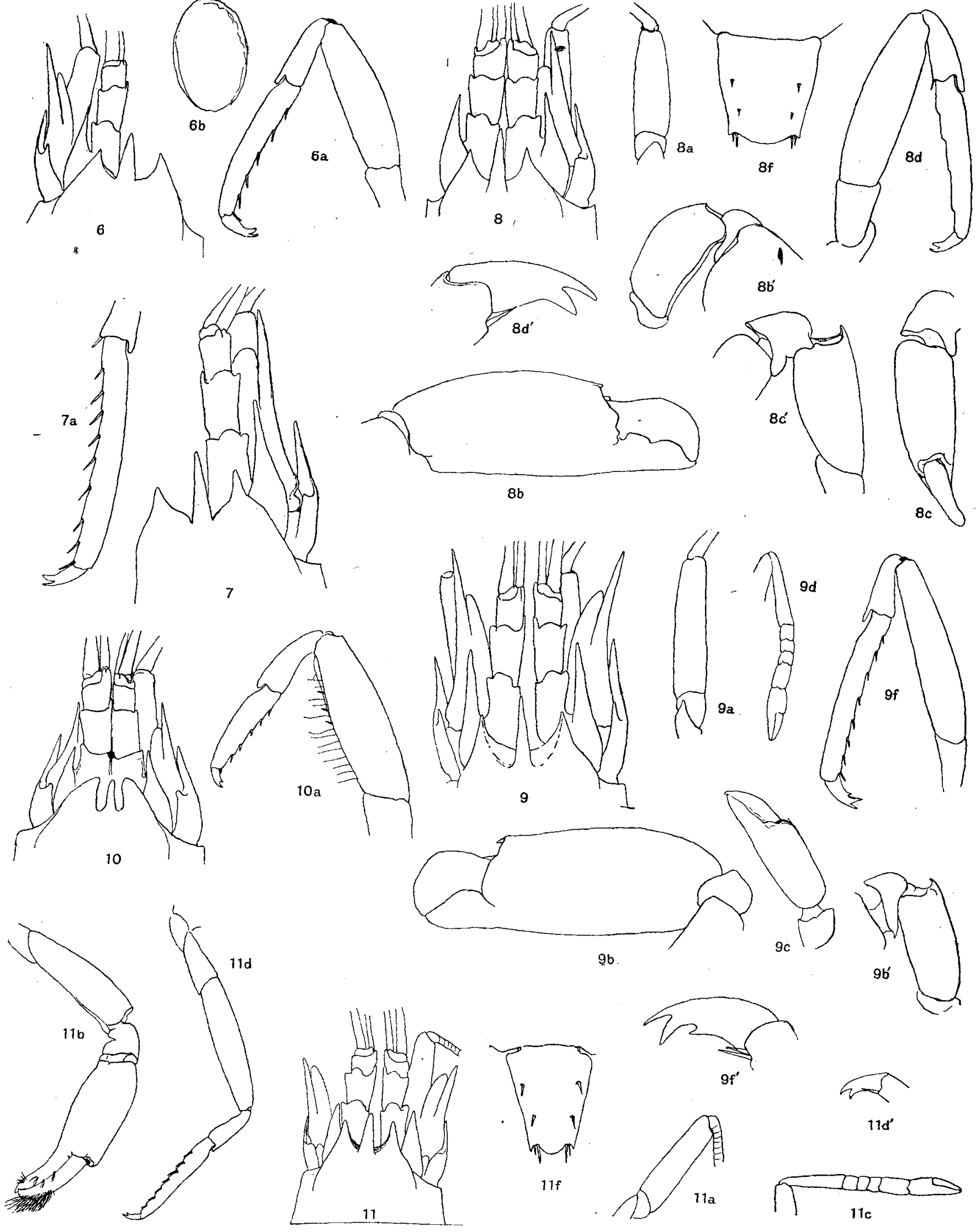

\section{ALPHEIDAE}


Percy Sladen Trust Expedition. (COUTIÈre)

TRANS. LINN. SOC. SER. 2, ZOOL. VOL. XVII. PL. 62
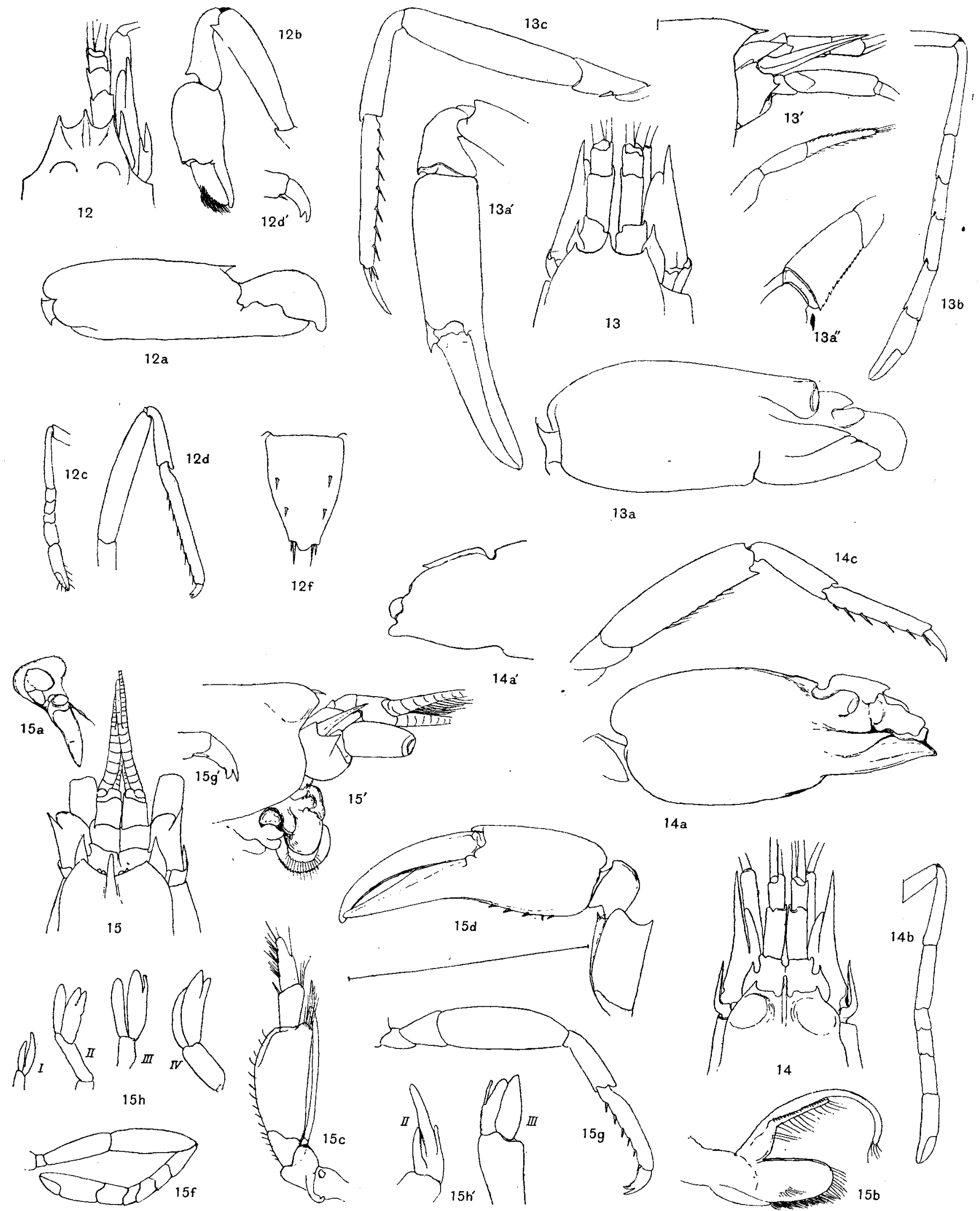

ALPHEIDAE 
Percy Sladex Trust Expedition.

(Coutière)

TRANS. LINN. SOC. SER. 2, ZOOL. VOL. XVII. PL 63
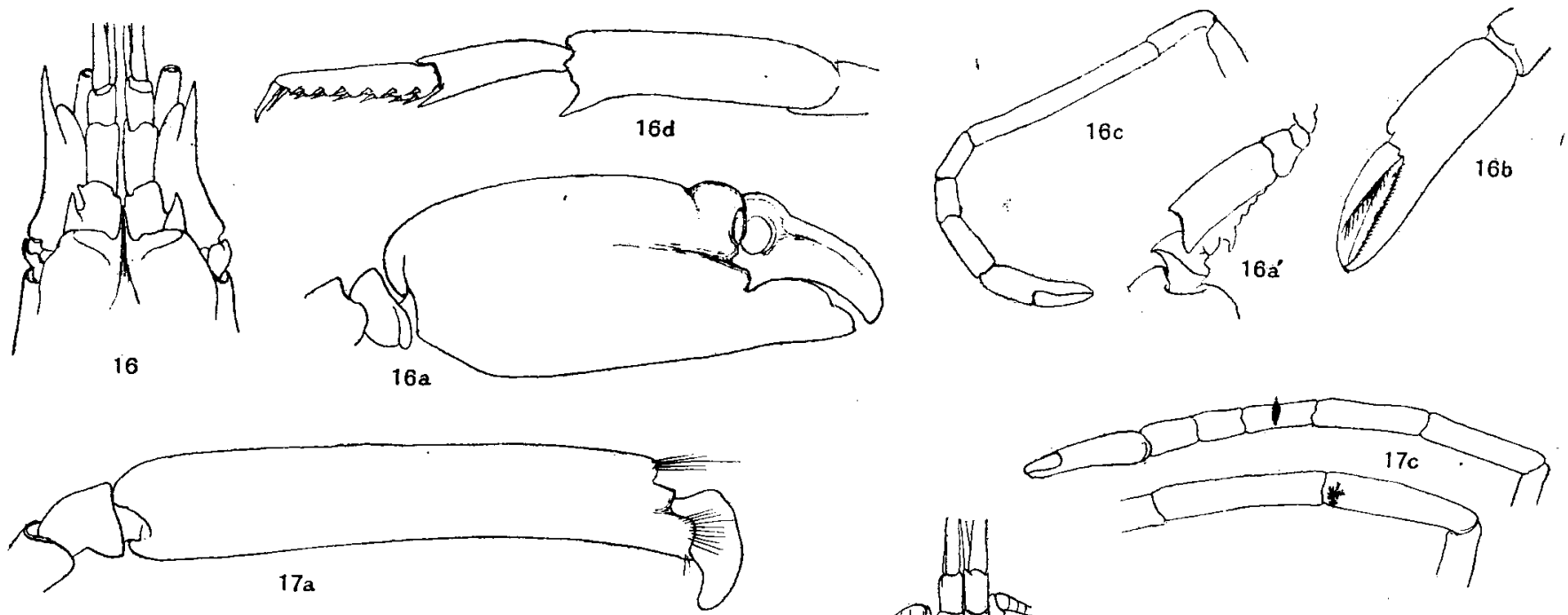
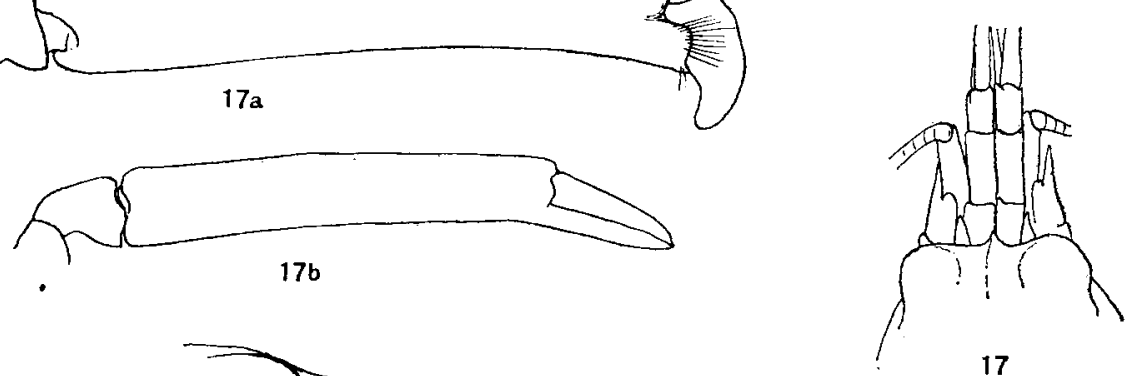

17
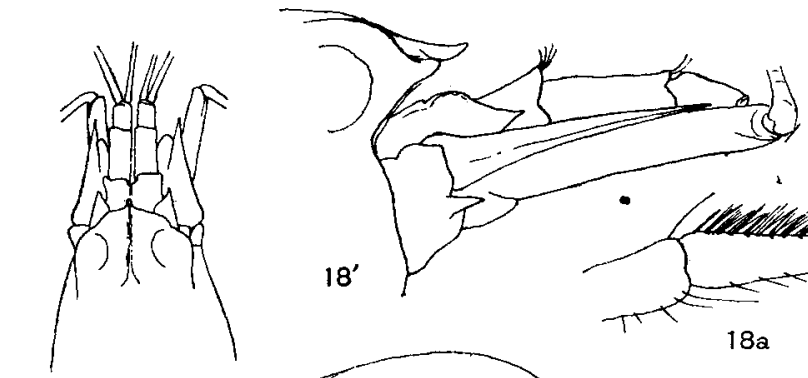

$18^{\prime}$
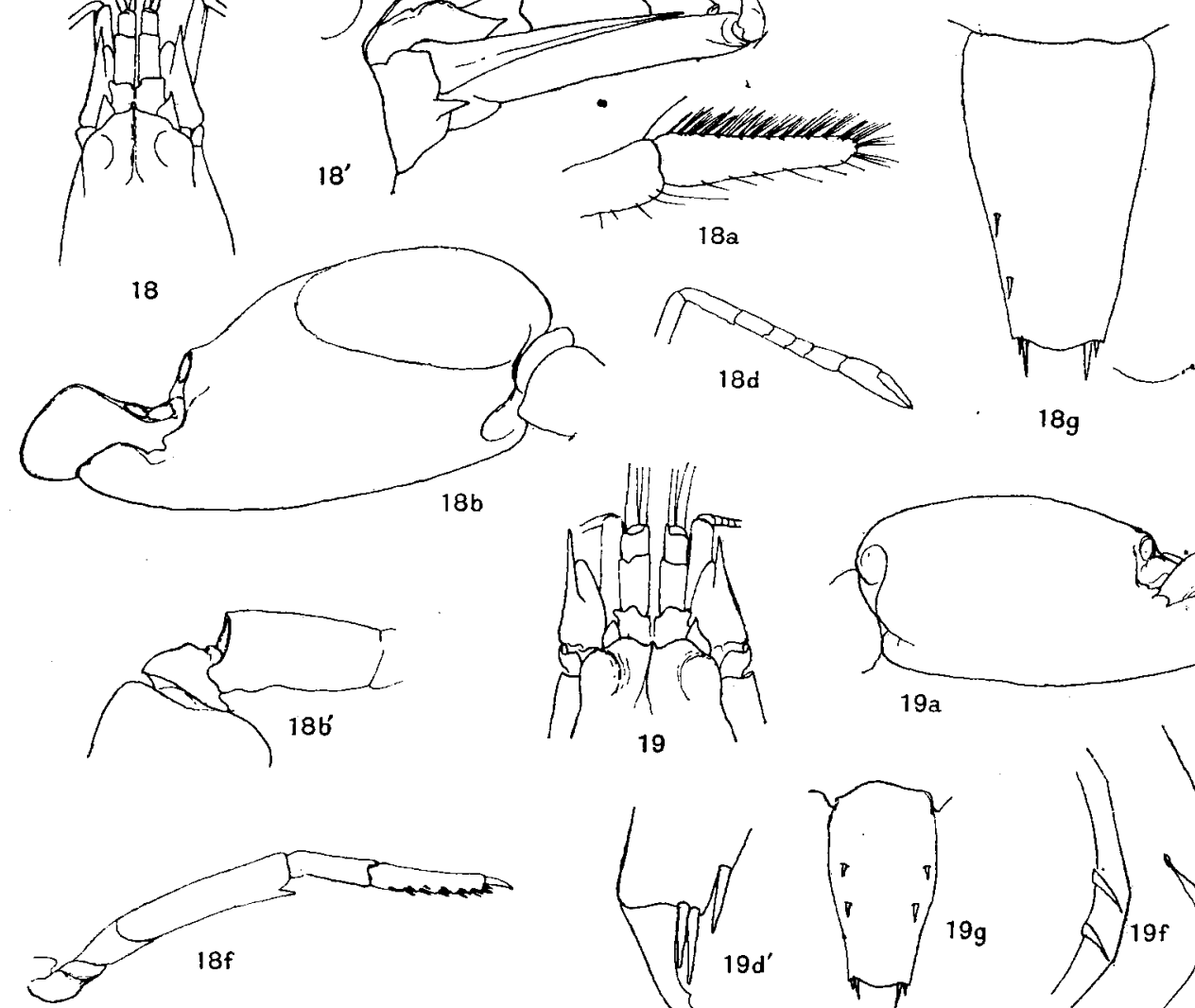

$18 \mathrm{~g}$
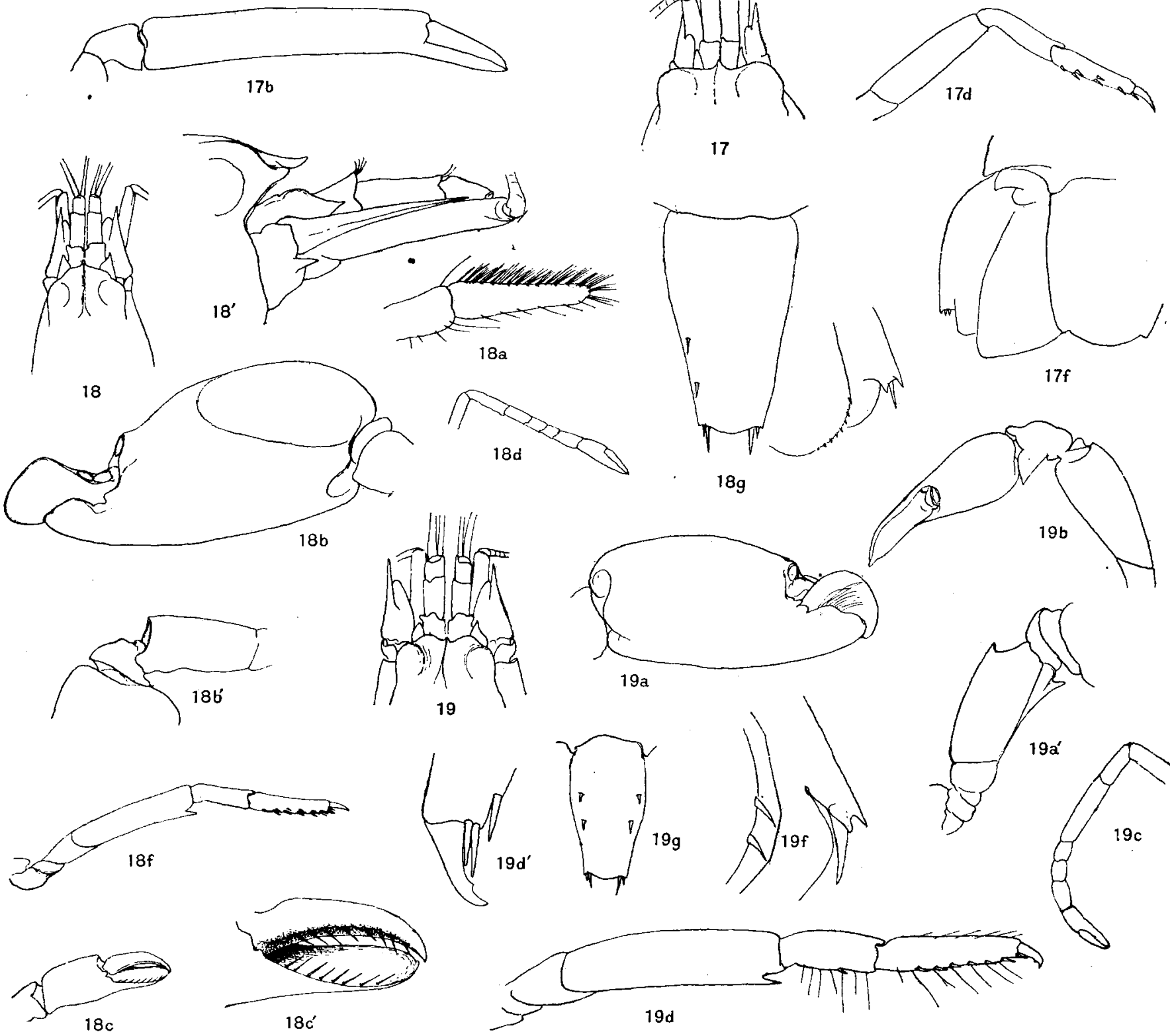

$18 c^{\prime}$
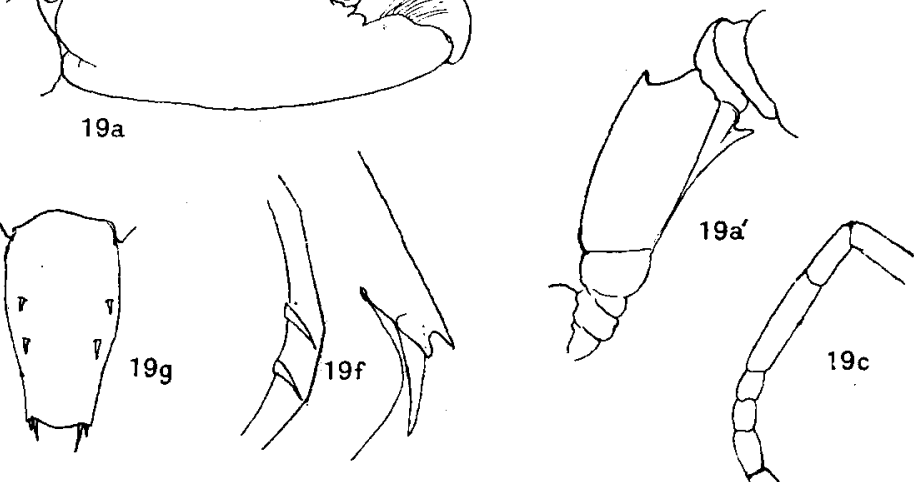

ALPHEIDAE 
Precy Sladex Trugi Expedition. (Coutière)

TRANS. LINN. SOC. SER. 2, ZOOL. VOL. XVII. PL. 64
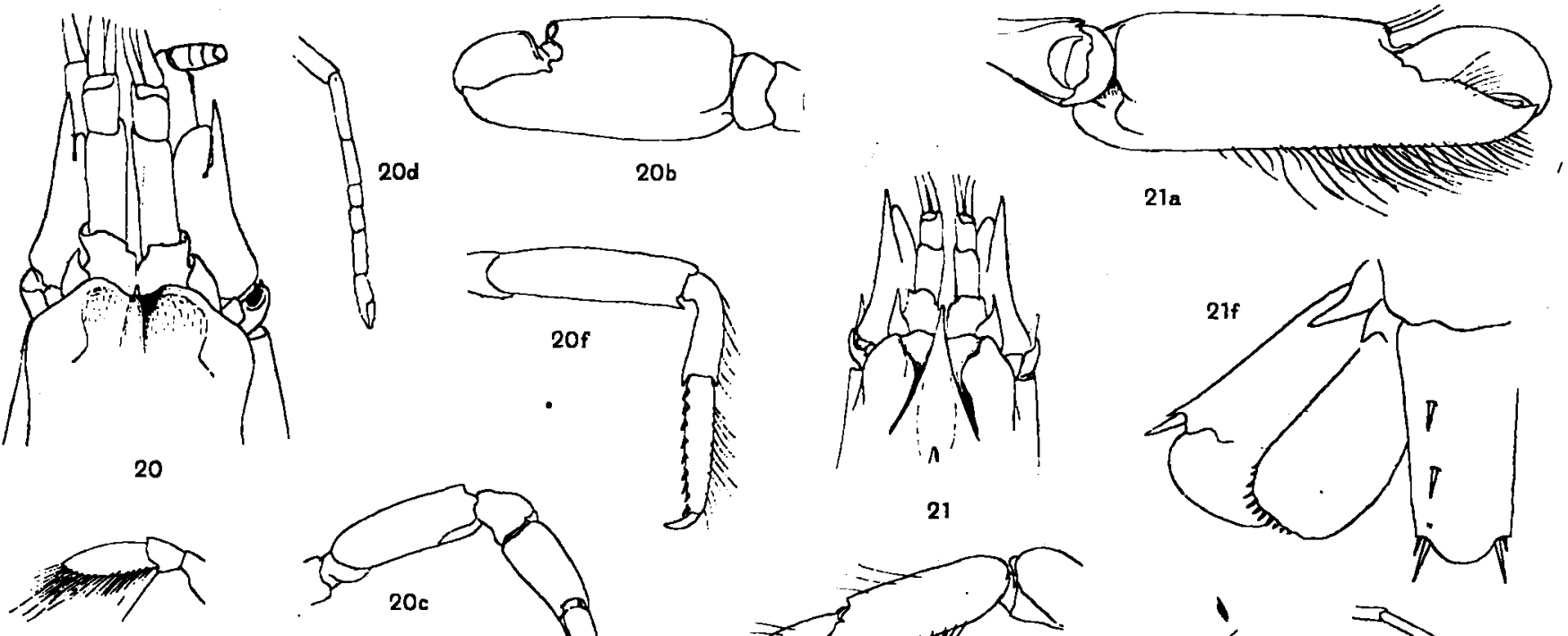

$20 \mathrm{a}$
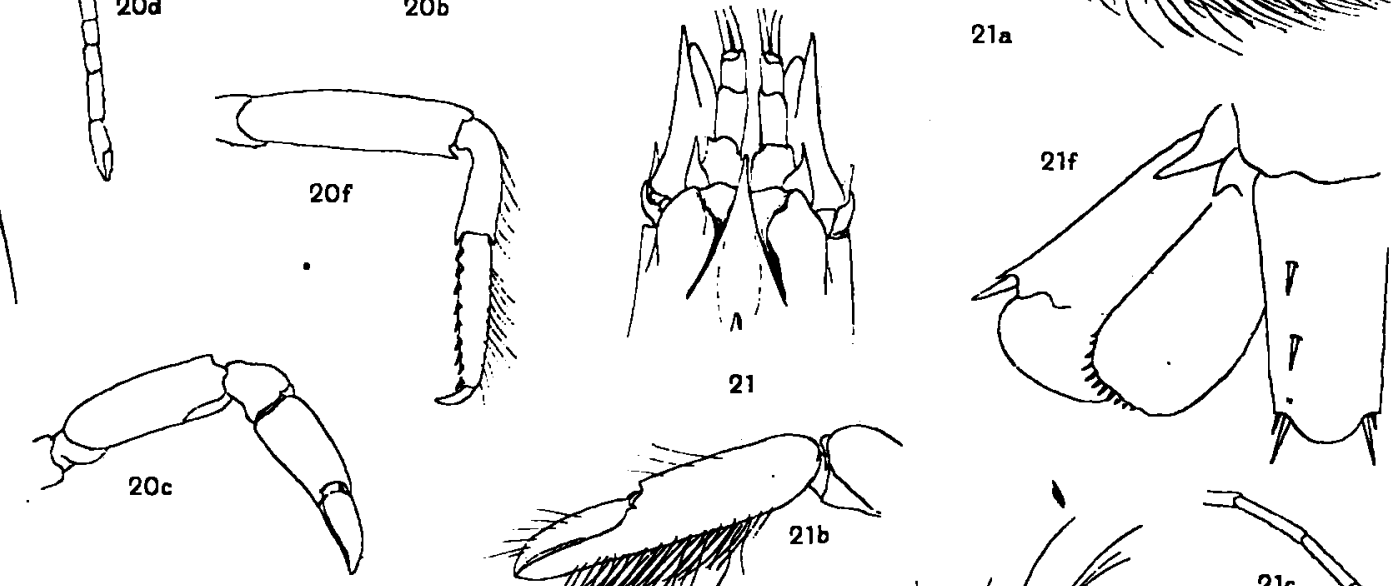

21
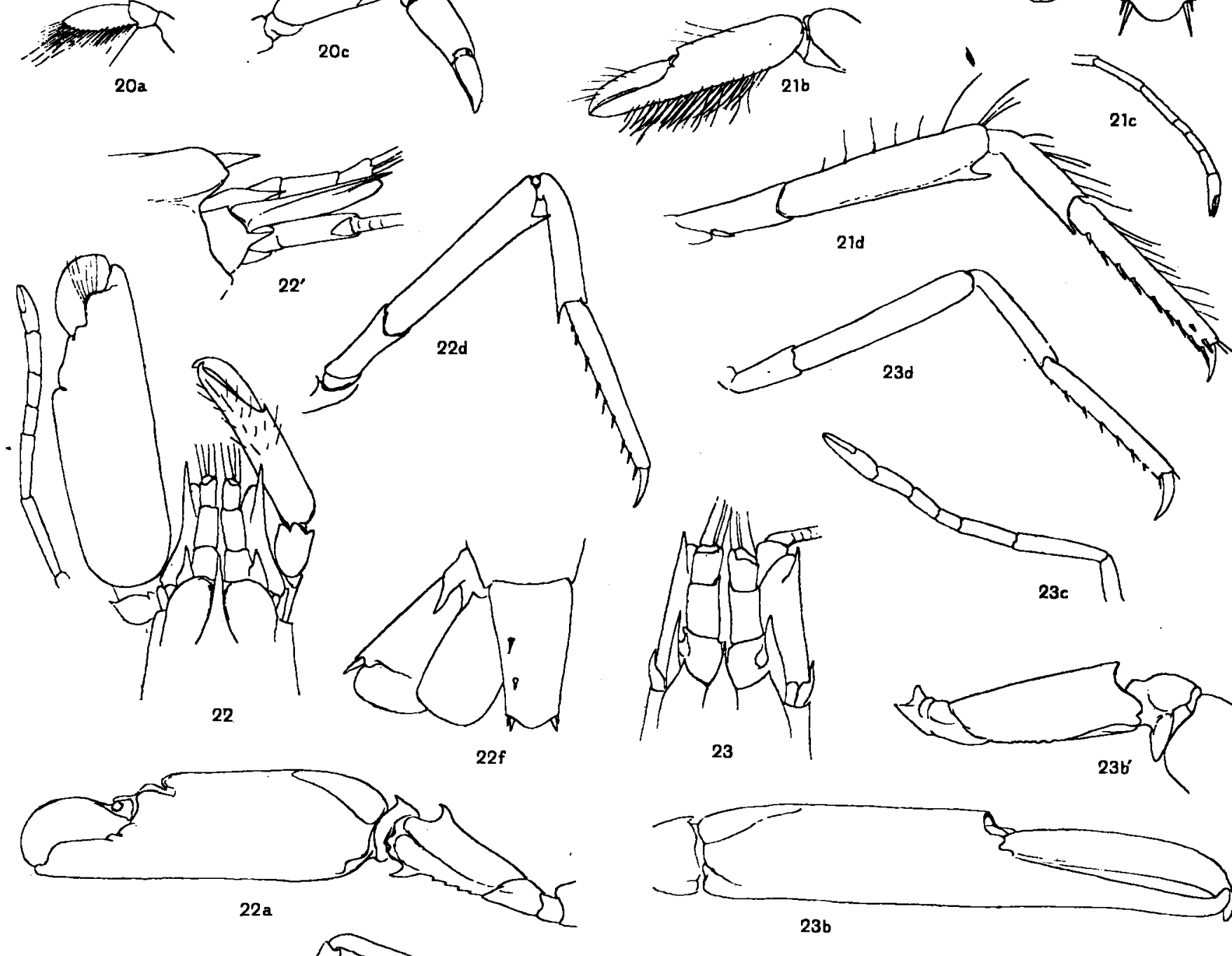

1d
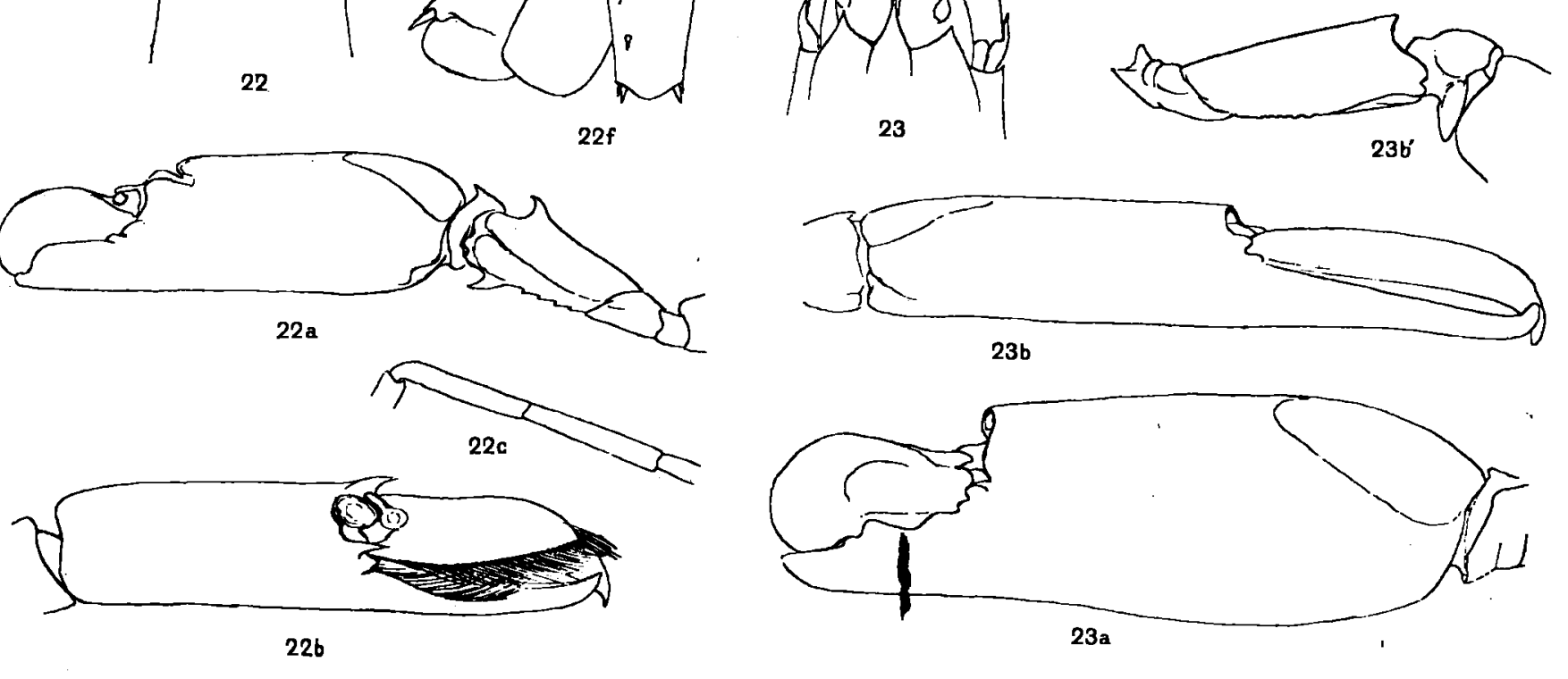

ALPHEIDAE 